\title{
Renforcement des capacites villageoises: Comment 23 villages s'initient aux Droits Humains et abandonnent la pratique de l'excision au Burkina Faso
}

\author{
Nafissatou J. Diop \\ Population Council \\ Edmond Bagde \\ Population Council \\ Djingri Ouoba \\ Molly Melching
}

Follow this and additional works at: https://knowledgecommons.popcouncil.org/departments_sbsr-rh

Part of the Community-Based Learning Commons, Community-Based Research Commons, Gender and Sexuality Commons, International Public Health Commons, Maternal and Child Health Commons, Public Health Education and Promotion Commons, and the Women's Health Commons How does access to this work benefit you? Let us know!

\section{Recommended Citation}

Diop, Nafissatou J., Edmond Bagde, Djingri Ouoba, and Molly Melching. 2003. "Renforcement des capacites villageoises: Comment 23 villages s'initient aux Droits Humains et abandonnent la pratique de l'excision au Burkina Faso." Dakar: Population Council. 


\section{SUMMARY}

One of the foremost priorities at the international level in recent years has been to improve women's reproductive health and welfare. The government of Burkina Faso, in its response to this issue, clearly expressed its will to work towards improved reproductive health in general, and to fight against female genital cutting in particular.

This is the background for the Community Based Education Program implemented by Mwangaza Action, with a view to improving women's reproductive health and putting an end to female genital cutting. Mwangaza Action replicated the TOSTAN Program also named the Village empowerment program (VEP) that resulted in public declarations of abandonment of female genital cutting in 938 village communities in Senegal.

In Burkina Faso, the Community Based Education Program involves 23 villages in the Zoundweogo province and in the departments of Bere and Binde. It is a holistic program which includes four modules relating to several areas of community welfare: education in human rights, problem solving processes, hygiene and disease prevention, women's health. These modules are taught through 64 sessions during which the participants (men and women) discuss to find solutions to several problems faced by their communities.

During implementation of this program, positive change has been noted in the beneficiary villages. The promotion of reproductive health and human rights by the communities and the regular activities of public hygiene have been observed. Also, these villages expressed their opposition to violence against women. Lastly, the program improved the population's knowledge and changed their views on women's role in community development activities. The utilization of health services, antenatal consultations, family planning and children care showed significant increases.

The 23 communities finally made a public declaration of abandonment of female genital cutting practices in front of 5000 villages, religious, traditional and political leaders, program managers, from government, NGOs and international organizations and medias.

This document is a synopsis of the entire process implemented to allow for replication of the TOSTAN Program and the results achieved in the social and cultural context of Burkina Faso. 
TABLE DES MATIERES

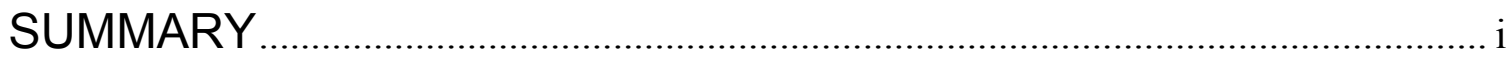

TABLE DES MATIERES ................................................................................ ii

REMERCIEMENTS ......................................................................................... ii

LISTE DES ABREVIATIONS ....................................................................... iv

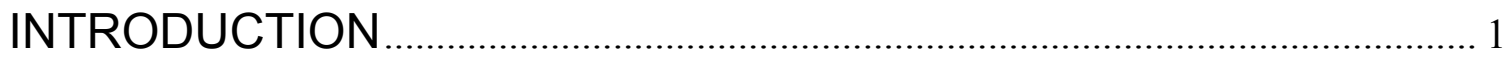

ORIENTATIONS DU PROGRAMME ……........................................................ 1

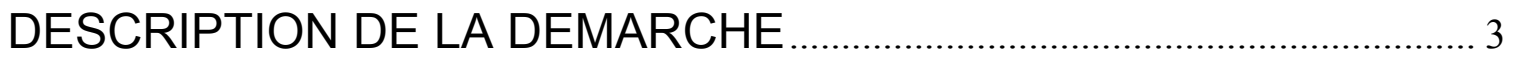

Phase de Préparation........................................................................................................... 3

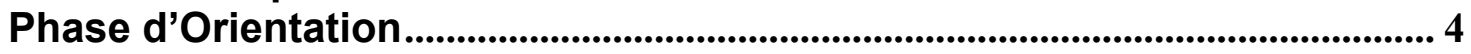

Phase d'Organisation ........................................................................................................ 10

Phase de Mise en Oeuvre ............................................................................12

Phase de Suivi et d'Evaluation.......................................................................... 18

DYNAMIQUE COMMUNAUTAIRE ……....................................................... 18

RESULTATS DE L'INTERVENTION............................................................. 20

ENGAGEMENT DES 23 VILLAGES DEVANT 5000 PERSONNES.. 23

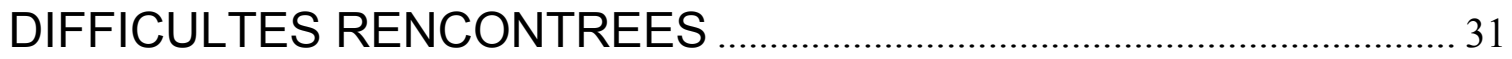

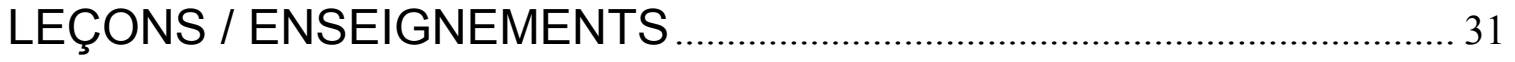

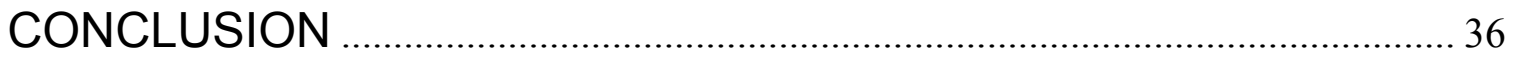




\section{REMERCIEMENTS}

MWANGAZA ACTION adresse ses remerciements à tous ceux qui l'ont soutenu techniquement et financièrement dans le cadre de mise en œuvre de ce programme d'éducation à base communautaire : le Comité Nationale de Lutte Contre la Pratique de l'Excision, l'ONG TOSTAN, le Programme Frontières en Santé de la Reproduction du Population Council, l'USAID, la GTZ (Promotion des Initiatives pour l'Eradication des Mutilations Génitales Féminines).

Nous exprimons nos sincères remerciements à TOSTAN, sans qui cette expérience n'aurait pas pu avoir lieu. En effet, TOSTAN a gracieusement mis à la disposition de Mwangaza leur curriculum, et tous les outils pédagogiques nécessaires à la mise en œuvre du programme. TOSTAN a aussi assuré la formation et l'encadrement des agents de Mwangaza Action tout le long du programme.

Nous exprimons notre gratitude aux Dr Nafissatou Diop, Dr Inge Baumgarten, et Mme Pascaline Sebgo qui nous ont encouragé à écrire sur cette expérience.

Ce programme n'aurait pas été réellement effectif sur le terrain sans le dévouement et l'effort conjugué de tous les agents de développement, en l'occurrence les facilitateurs communautaires, les superviseurs et les coordonnateurs. Nous formulons nos remerciements à toute cette équipe. Nous pensons qu'elle trouvera dans ce travail la récompense de ses efforts.

Nous ne saurions oublier les populations des départements de Béré et Bindé dans la province du Zoundwéogo. Leur accueil, leur disponibilité et leur participation aux activités ont été déterminants.

Notre reconnaissance va à l'endroit des autorités administratives et leaders communautaires de la zone d'intervention pour leur soutien et implication au processus de mise en œuvre de ce programme.

Nous remercions enfin M. Edmond Bagdé pour ses feedback et la mise en forme du présent document. 


\section{LISTE DES ABREVIATIONS}

CIPD Conférence Internationale sur la Population et Développement

CSPS Centres de Santé et de Promotion Sociale

CGV Comité Villageois de Gestion

MGF Mutilation Génitale Féminine 


\section{INTRODUCTION}

La Conférence Internationale sur la population et le Développement (CIPD) tenue au Caire (Egypte) en 1994 a été un déclic en matière de santé de la reproduction. Les 174 pays ayant participé à cette rencontre ont pris un engagement international visant à améliorer la santé reproductive et à protéger les droits reproductifs.

Le but visé par la politique de santé de la reproduction est de contribuer à l'amélioration de l'état de santé et du bien-être des populations dans la perspective d'un développement humain durable. La question de la santé de la reproduction est perçue comme un droit essentiel pour les enfants, les jeunes, les femmes et les hommes. Elle a un impact certain sur le développement humain durable.

Afin de contribuer à l'amélioration de la santé sexuelle et reproductive des enfants, des jeunes, des femmes et des hommes, MWANGAZA ACTION a mis en œuvre un programme d'éducation à base communautaire dans la province du Zoundwéogo au Burkina Faso. C'est une réplication du programme de l'ONG sénégalaise TOSTAN. En effet, TOSTAN a développé un curriculum et une approche de travail avec la communauté qui lui a permis d'exécuter des programmes ayant abouti à des déclarations publiques d'abandon de la pratique de l'excision dans 938 communautés sénégalaises.

Le programme d'éducation à base communautaire se révèle par son caractère singulier dans la panoplie des approches de développement menés au Burkina du fait de l'accent mis sur les Droits Humains. Il a été exécuté dans 23 villages de la province du Zoundwéogo. L'accent a été mis sur la promotion de la santé de la reproduction et des Droits Humains pour lutter contre les violences faites aux femmes avec une considération particulière pour l'excision.

Le programme a été cofinancé par l'USAID, dans le cadre du Programme «Frontières en Santé de la Reproduction» exécuté par le Population Council et la GTZ dans le cadre du «Projet Supra régional de lutte contre les Mutilations Génitales Féminines».

Le présent document décrit la démarche suivie pour la mise en oeuvre du programme.

\section{ORIENTATIONS DU PROGRAMME}

Le but du programme d'éducation à base communautaire est de contribuer à l'amélioration de la Santé de la Reproduction de la population des aires de santé de Béré, Bindé et Sondré dans le district sanitaire de Manga, province du Zoundwéogo au Burkina Faso. Son objectif général est de mettre à la disposition des décideurs, des partenaires financiers, responsables des Associations et ONG, une initiative destinée à renforcer le programme de santé et d'éliminer les mutilations génitales féminines (MGF) au Burkina Faso.

Les objectifs spécifiques assignés au programme sont : 
a) renforcer les capacités des populations sur des enseignements et apprentissages relatifs aux droits humains, au processus de résolution des problèmes, à l'hygiène et à la santé de la femme;

b) accroître la mobilisation des populations sur les questions de santé de la reproduction en organisant des rencontres et discussions publiques.

Concernant les résultats attendus, au terme du programme, les femmes et les hommes doivent acquérir :

- des connaissances sur les Droits Humains et sur la manière de les appliquer pour améliorer leurs conditions ;

- des aptitudes à résoudre les problèmes de la famille et de la communauté;

- des informations techniques relatives à l'hygiène et à la prévention des maladies ;

- des connaissances sur le développement et les fonctions des organes du corps humain ;

- des informations sur les méthodes de promotion de la santé à toutes les étapes du développement du corps humain ;

- des connaissances sur les pratiques traditionnelles néfastes pour la santé et les stratégies pour y mettre un terme ;

- des aptitudes à communiquer afin de partager les informations avec les autres membres de la communauté et les autres villages de la zone ;

- une motivation à œuvrer à la recherche des solutions communautaires aux problèmes du village ;

- une confiance renouvelée en leur capacité à susciter des changements sociaux positifs.

En terme de stratégies, cinq aspects caractérisent le programme :

$>$ Approche basée sur les Droits Humains

$>$ Classes homogènes et des séances un jour sur deux, selon les horaires choisis par la population

$>$ Système de parrainage où chaque auditeur adopte une personne de sa communauté pour partager avec elle les informations qu'elle reçoit dans la classe

$>$ Facilitateurs installés dans les villages d'intervention

$>$ Programme à double volet : intervention et recherche 
Le paquet pédagogique du programme comprenait quatre modules, notamment :

a) Les Droits Humains. L'éducation aux Droits Humains permet aux participants des classes, en particulier, et à la population en général, de comprendre et de défendre leurs droits par rapport à la santé et à leur intégrité physique. Elle permet une transformation sociale positive et pacifique qui conduit à un état de bien-être physique, social et mental.

b) Le processus de résolution des problèmes. Les participants s'initient à un processus simple de résolution des problèmes qui les aident à mieux identifier et analyser leurs problèmes, trouver des solutions qui s'adaptent à leurs situations et à les mettre en œuvre au plan individuel, familial et communautaire.

c) Hygiène et prévention des maladies. Les participants acquièrent des informations leur permettant d'entreprendre des actions en faveur de la santé de la femme et de la communauté en général.

d) Santé de la femme. Les participants ont des informations sur le fonctionnement et les rôles des différents organes du corps humain. C'est la promotion de la santé de la femme qui est visée par un encouragement à adopter des comportements sains.

Après 18 mois d'intervention dans les villages pour la mise en œuvre de ces quatre modules, voici la démarche qui a été suivie sur le terrain pour la promotion de la santé de la reproduction et des Droits Humains.

\section{DESCRIPTION DE LA DEMARCHE}

La mise en place du programme d'intervention a nécessité le développement de la démarche de mobilisation sociale. Cette démarche s'articule autour de cinq phases : la phase préparatoire, la phase d'orientation, la phase d'organisation, la phase de mise en œuvre et la phase d'évaluation.

\section{Phase de Préparation}

Le programme que MWANGAZA ACTION a mis en œuvre au Burkina Faso est basé sur le programme de l'ONG sénégalaise TOSTAN. Cette phase s'articule autour des actions d'appropriation du programme TOSTAN par l'équipe de MWANGAZA ACTION. A cet effet, plusieurs actions ont été développées : 


\section{Traduction des documents pédagogiques}

Les différents documents des quatre modules à enseigner ont été traduit en langue mooré. Il s'agit du guide numéro 1 qui comporte le module éducation aux Droits Humains et le module processus de résolution des problèmes, le guide numéro 2 comportant le module hygiène et prévention de la maladie, et la boîte à images qui accompagne le module santé de la femme.

\section{Formation de l'équipe d'encadrement}

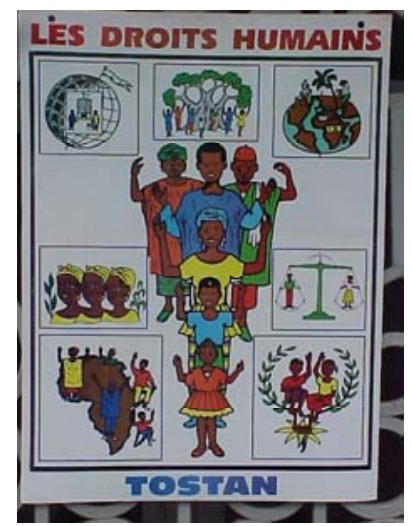

Une équipe d'encadrement, composé de deux chargés de programme de MWANGAZA ACTION et de trois superviseurs, a été constituée. Cette équipe a séjourné pendant un mois à Thiès au Sénégal. L'équipe de MWANGAZA ACTION a eu des échanges avec les homologues du Sénégal où elle a été formée par l'ONG TOSTAN. Cela a permis aux formateurs Burkinabé de comprendre l'articulation du programme d'éducation à base communautaire et de s'approprier le programme TOSTAN.

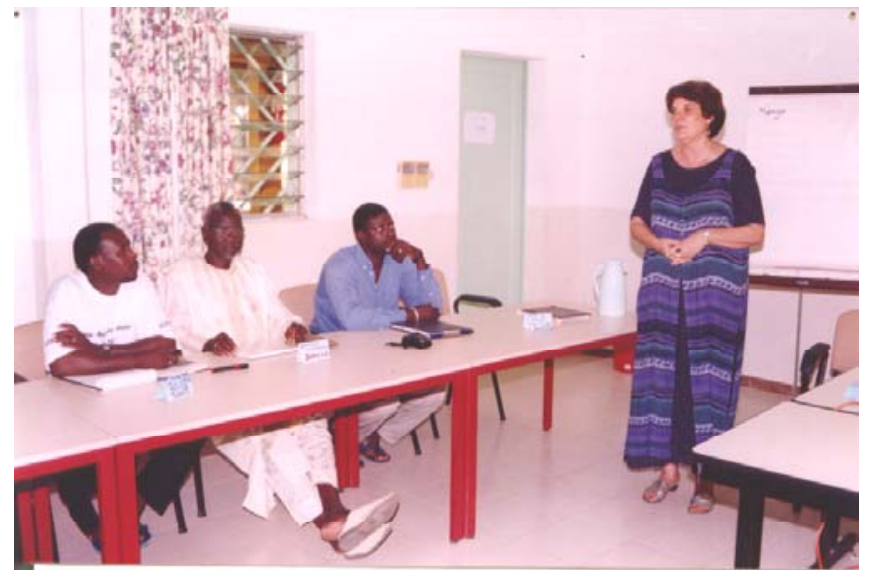

Formation des formateurs à Thiès au Sénégal. On reconnaît ici debout Molly Melching de l'ONG TOSTAN.
L'équipe du Burkina Faso a eu l'opportunité de participer à la cérémonie de déclaration publique de promotion de la femme dans la localité de Aéré-lao puis celle de Karcia. Cela a permis aux formateurs Burkinabé de s'imprégner du processus organisationnel et d'animation d'une cérémonie de déclaration publique.

\section{Phase d'Orientation}

Cette phase a été consacrée à la présentation, discussion, information des différentes parties prenantes sur les grandes lignes du programme d'éducation à base communautaire. Les différentes étapes de cette phase sont :

\section{Recrutement des facilitateurs communautaires}

Vingt trois (23) facilitateurs communautaires ont été recrutés pour la mise en œuvre du programme sur la base des critères suivants : le niveau d'instruction (niveau terminal et plus), la maîtrise de l'outil linguistique ( mooré dialecte dominant), la motivation à travailler en milieu rural. Cette équipe, composée de 13 femmes et 10 hommes, a travaillé de décembre 2000 en juin 2001. A la suite de la suspension des activités du programme 
pendant la saison des pluies, 12 facilitateurs communautaires n'ont plus voulu poursuivre les activités de mise en œuvre du programme. Ceux-ci ont alors été remplacés par 12 autres facilitateurs communautaires. Ce qui a permis de reconstituer la nouvelle équipe pour la mise en œuvre des activités du programme d'octobre 2001 à mars 2002. Cette deuxième équipe ainsi constituée, comprenait 10 femmes et 13 hommes.

La formation des ressources humaines est une tâche très importante dans le processus de renforcement des capacités des populations. Par conséquent, il serait préférable de garantir l'emploi du personnel exécutant du programme pour toute la durée du processus, afin de s'assurer de la continuité des actions par les mêmes acteurs. En effet, une interruption momentanée entraîne souvent le départ du personnel à la recherche d'autres opportunités. Ce qui est préjudiciable au programme, obligé de procéder à un autre processus de recrutement et de formation.

\section{Orientation et information des facilitateurs sur les grandes lignes du Programme d'Education à Base Communautaire}

Un atelier de deux jours a été organisé à l'intention des facilitateurs communautaires. Trois objectifs étaient visés :

- orienter les membres de l'équipe de mise en œuvre sur le programme afin qu'ils aient une vision claire de son contenu et du rôle qui est attendu de Mwangaza dans la mise en œuvre des activités ;

口 préparer le stage d'immersion des Facilitateurs dans les communautés ;

- maîtriser la démarche de présentation du programme aux populations.

L'atelier a servi de cadre pour une connaissance mutuelle entre les personnes chargées de la mise en œuvre du projet à savoir les facilitateurs communautaires, les superviseurs et les coordonnateurs. L'atelier a été animé avec les techniques actives de formation. Des exercices de team building ont été développés pour renforcer la cohésion et l'esprit d'équipe entre tous les acteurs du programme.

\section{Stage d'immersion des facilitateurs}

Afin de les préparer à leur nouveau rôle d'appui à la communauté d'une part, et de créer un cadre de connaissance mutuelle entre les deux entités et le programme d'autre part, il y a eu un stage d'immersion au sein des communautés partenaires du programme. Ce processus de socialisation a consisté en un séjour de trois semaines dans les villages. C'est une occasion offerte au personnel nouvellement recruté comme facilitateur communautaire de se mettre en contact avec la communauté afin de réapprendre un certain nombre de valeurs (simplicité, acceptation des autres tels qu'ils sont etc.) pouvant les aider dans le travail avec les communautés. Les objectifs assignés à un tel stage d'immersion étaient :

- Etablir des relations de confiance avec la communauté et ses responsables dans le but de bâtir une action pérenne. 
- Connaître la population des localités (villages) où interviendra le programme, les différentes autorités et se faire connaître en tant qu'individu mais aussi en tant qu'agent du développement.

- Donner aux facilitateurs communautaires une connaissance et une bonne photographie de la zone d'intervention du projet et faciliter leur intégration.

- Collecter des informations de base devant permettre aux techniciens d'appui à la communauté de faire ses premières analyses de la situation de la communauté en matière de santé maternelle et néonatale.

Pour cela, chaque Facilitateur a été installé dans la communauté après des négociations avec les responsables du village. Ensuite les responsables du village, après l'aboutissement de la négociation, ont identifié une famille ou un local dans lequel le Facilitateur allait être hébergé.

Durant ce séjour villageois de trois semaines, les Facilitateurs Communautaires se sont intéressés à toutes les personnes (personnel de santé et membres des comités de gestion, responsable administratif villageois, chefs de villages et personnes influentes, leaders religieux, responsables d'associations et groupements de femmes et d'hommes etc.) ou structures dans la communauté qui leur permettront de réaliser leurs objectifs. La méthode de travail utilisée durant ce stage d'immersion était :

1. Observation des réalités du milieu et participation aux activités villageoises ;

2. Interviews et entretiens avec les différents groupes cibles identifiés au sein de la communauté

Au terme de trois semaines de séjour dans les villages, les aspects suivants étaient comptabilisés comme résultats :

1. Connaissance de la population en terme de pratiques, d'organisation, d'us et coutumes ;

2. Apprentissage de la «logique communautaire »;

3. Apprentissage de la langue et du langage de la communauté ;

4. Apprentissage d'une autre façon de vivre ;

5. Début d'apprentissage de la situation de la communauté en relation avec les orientations du programme d'éducation à base communautaire. 
Le stage d'immersion a permis aux communautés et aux facilitateurs de bâtir des relations basées sur la confiance mutuelle, le respect de l'autre et le partage des responsabilités.

\section{Rencontres informelles avec les autorités locales, administratives et techniques}

Des rencontres informelles ont été organisées avec les autorités administratives (Secrétaire Général de la province du Zoundwéogo, Préfets des départements de Béré et Bindé), les responsables des services techniques (Responsable Provincial de l'Action Sociale et de la Solidarité Nationale, Médecin-chef de District), les responsables des formations sanitaires périphériques, les Comités de Gestion, les Responsables Administratifs de Village, les responsables coutumiers (chefs de villages, de quartiers ou de secteurs), les responsables religieux et autres personnes influentes de la zone du programme.

Ces rencontres ont consisté à discuter de façon informelle avec ces différents responsables sur les grandes lignes du programme, les objectifs et la démarche de mise en œuvre. Ces actions ont abouti à la planification de rencontres / réunions formelles avec les responsables / représentants communautaires.

\section{Rencontre de présentation et discussion des grandes lignes du programme avec les représentants des villages}

Les réunions planifiées au moment des contacts informels ont permis de regrouper tous les responsables / leaders communautaires de chaque village (y compris les membres des Comités de Gestion des Formations Sanitaires) au niveau des centres de santé et de promotion sociale (CSPS). Ont participé aux rencontres les personnes suivantes :

- les agents de santé communautaires ;

- les responsables religieux ;

- les chefs coutumiers ;

- les responsables administratifs de village ;

- les membres des comités de gestion des centres de santé ;

- les agents de santé des CSPS ;

- les responsables de certains services techniques (enseignement de base, environnement, agriculture etc.) ;

- les responsables de groupements villageois ;

- les responsables d'ONG ;

- les préfets.

Au total 144 personnes ont participé aux trois rencontres. Les discussions ont porté sur les objectifs du programme, son concept, son contenu, ses stratégies, ses implications, les rôles et responsabilités des différents partenaires (MWANGAZA ACTION, Communauté), les attentes du programme vis-à-vis de chaque communauté et celles des communautés. 


\section{RESPONSABILITES DE CHAQUE COMMUNAUTE}

- Accueillir et héberger le facilitateur communautaire

- Déterminer le lieu et / ou construire un abri qui servira de salle de classe

- Mettre en place un comité de gestion

- Identifier les auditeurs et auditrices

- Gérer le matériel et les fournitures

- Encourager la participation des femmes

- Entretenir la classe

- Créer un cadre d'échange entre les participants au programme éducatif et la communauté

- Utiliser les apprentissages pour des actions concrètes dans la communauté

\section{RESPONSABILITES DE MWANGAZA ACTION}

- Assurer la formation des facilitateurs communautaires

- Assurer la mise en œuvre du programme en mettant à la disposition de chaque village un facilitateur pour les animations des classes, le suivi, et l'évaluation des activités du programme éducatif

- Mettre à la disposition de la communauté, le matériel et les fournitures nécessaires à l'animation des classes

- Assurer la mise en œuvre du volet recherche du programme en collaboration avec les autres partenaires dans le but de documenter le processus

Ces discussions ont été introduites par une saynète qui était axée sur le programme d'éducation à base communautaire. En rappel, la saynète est une technique active qui suscite la participation de tout le monde dans la discussion.

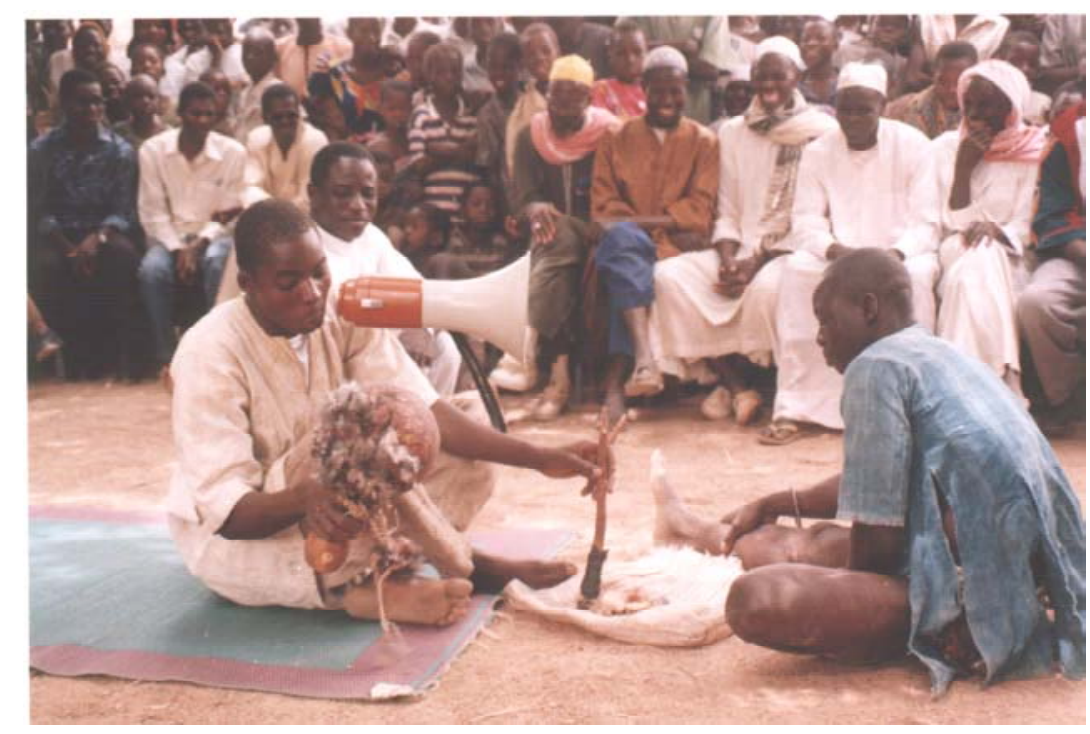

Une séance de saynète lors d'une rencontre inter villageoise 


\section{SAYNETE UTILISEE POUR PRESENTER LE PROGRAMME}

\section{Objectif :}

Permettre aux villageois de discuter des actions entreprises dans le village de LAAFIBALA en faveur de la santé de la reproduction des femmes et des jeunes filles. (La saynète se déroule en II tableaux avec 3 acteurs)

\section{Tableau I}

Ousmane, un aventurier revient dans son village LAAFIBALA qu'il a quitté depuis très longtemps. A LAAFIBALA, il constate des changements. Le village est propre, les infrastructures de santé et d'éducation existantes sont bien entretenues... Dans son commentaire (monologue), Ousmane fait ressortir le fait qu'il y ait beaucoup de femmes au dispensaire. II remarque que le dépotoir d'ordure devient un terrain de jeu ou un lieu de discussion. Il est émerveillé par tant de changements. Il se rend chez son ami d'enfance qui n'a pas quitté le village pour se renseigner.

\section{Tableau II}

Chez TIRAOGO, l'ami d'enfance, Ousmane est reçu avec beaucoup de joie (Lorsqu'il arrive chez son ami Tiraogo, sa femme lui donne de l'eau et veut s'en retourner et Tiraogo lui dit de ne pas partir parce qu'ils (ensemble) ont un étranger ; ce qui montre l'effort sur l'intégration des femmes dans les histoires familiales). Après les salutations, il se renseigne sur l'origine des changements qu'il a constatés dans le village. TIRAOGO et sa femme lui expliquent que les habitants de LAAFIBALA ont bénéficié de l'intervention d'un programme d'éducation de base (II est très important de préciser les orientations du programme, sa nature et son but). Les thèmes qui ont été développés dans les classes sont les Droits Humains, le processus de résolution des problèmes, l'hygiène et la santé de la femme. TIRAOGO et sa femme lui apprirent que ce sont les connaissances acquises durant les animations dans les classes qui leur ont permis d'entreprendre des actions pour le développement de la communauté en général et des femmes en particulier. OUSMANE très content de la nouvelle situation de son village demande à son ami de l'inscrire dans la classe afin qu'il puisse lui aussi bénéficier des mêmes enseignements. Sur ce, il prend congé de son ami.

\section{Questions à poser après la saynète}

1. Qu'est ce qui s'est passé dans le village de LAAFIBALA ?

2. Quel est le sujet développé dans la saynète ?

3. Comment le village de LAAFIBALA a-t-il évolué ?

4. Comment s'est organisée la communauté de LAAFIBALA ?

5. Qu'est ce qui a permis des changements dans le village de LAAFIBALA ?

6. Votre village veut-il ressembler à celui de LAAFIBALA ?

7. Qu'est ce que votre village veut faire ?

8. Etc.

Les responsables et leaders communautaires ont posé toutes les questions de clarification et de compréhension, afin de s'approprier le contenu du programme. La tâche assignée aux participants (responsables / représentants de village), après la rencontre, est de faire le compte rendu de la réunion dans leurs villages/quartiers respectifs. Il s'agit là en fait de la première étape de la stratégie de plaidoyer en direction des décideurs communautaires pour les acquérir à la cause du Programme. Une date est arrêtée avec chaque délégation 
villageoise pour la tenue de l'assemblée générale d'orientation et de discussion avec l'ensemble de la population du village.

\section{Assemblées générales villageoises pour présenter et discuter les grandes lignes du Programme}

Dans chacun des 23 villages, les populations - enfants, femmes et hommes - ont participé à une assemblée générale de présentation du programme. Au cours de ces assemblées, les participants ont discuté du contenu, des objectifs et des implications du programme pour chaque communauté. Ce sont les mêmes points discutés lors de la rencontre avec les leaders communautaires qui sont repris avec la population.

Ces grandes réunions, qui se sont tenues dans chaque village, avaient pour objet de toucher le plus grand nombre de personnes (femmes, enfants et hommes.). Environ 2071 personnes (841 femmes, 1005 hommes et 225 enfants) ont pris part aux rencontres tenues dans l'ensemble des villages. Ces rencontres ont aussi permis de recueillir les réactions des populations par rapport à leurs attentes mais aussi par rapport aux implications pour les villages qui accueillent le programme.

\section{Rencontre de feedback avec les représentants des villages}

Une rencontre avec les délégués de chaque village a eu lieu au niveau de chaque formation sanitaire. Elle se tient souvent après les rencontres villageoises. Chaque délégation villageoise fait le point du déroulement de l'assemblée générale de son village. Les points d'ombre relevés par les villages sont débattus.

Chaque délégation a manifesté officiellement son adhésion aux actions du programme. Cet acte constitue l'engagement libre du village à participer au programme et à respecter / honorer les rôles et responsabilités durant l'exécution des activités. C'est la première étape dans le partage des responsabilités entre les parties prenantes.

\section{Phase d'Organisation}

La phase d'organisation comprend toutes les actions rentrant dans le cadre de l'organisation de chaque communauté pour accueillir le programme. Les actions entreprises durant cette phase étaient :

\section{Mise en place des Comités Villageois de Gestion}

Le comité villageois de gestion est la structure la plus importante dans le processus de mise en oeuvre du programme dans les villages. Il joue le rôle d'intermédiaire entre le village et le programme. Pour mettre en place le Comité Villageois de Gestion dans chaque village, les leaders de chaque village ont :

- analysé les rôles du Comité Villageois de Gestion (CVG),

- établi des critères clairs et précis de sélection, 
- déterminé les compétences nécessaires pour assurer ces fonctions.

Sur la base de ces critères, chaque village a alors désigné les membres de son Comité de Gestion.

\section{CRITERES DE SELECTION DES MEMBRES DES COMITES VILLAGEOIS DE GESTION}

- Etre disponible

- Etre honnête

- Etre dynamique / Assidue aux travaux d'intérêt commun

- Etre capable de défendre les intérêts du village

- Etre respecté et respectueux

- Aimer le travail et savoir pardonner

- Avoir une situation sociale (s'entendre avec les gens - exercer une autorité, avoir un âge assez avancé)

- Savoir lire et écrire dans la langue mooré

- Ne pas être très mobile (voyages), mais se déplacer facilement

- Etre soucieux de la chose publique

- Associer les vieux et les jeunes dans le comité

- Tenir compte des quartiers du village en sélectionnant les membres

Au total 23 comités villageois de gestion ont été mis en place et comprennent 44 femmes et 154 hommes, soit un total de 198 personnes.

\section{TACHES DES COMITES VILLAGEOIS DE GESTION}

\section{Gestion du matériel :}

- Réceptionner le matériel destiné aux classes

- Entretenir la classe et le matériel

\section{Mobilisation de la communauté :}

- Motiver la population à participer au programme éducatif

- Organiser avec les participants des activités en dehors de la classe

- Organiser les réunions villageoises et inter-villages

- Résoudre les éventuels conflits

- Donner son avis sur le contenu du programme

\section{Identification des critères et choix des auditrices et auditeurs}

Les auditrices et les auditeurs sont les personnes qui participent à l'animation des séances dans les classes. Pour la sélection de ces personnes que l'on peut appeler « candidats à l'innovation », les membres du comité villageois de gestion et les leaders de chaque village se sont retrouvés et ont réfléchi sur le programme, sur ce que l'amélioration des connaissances à travers les auditrices et les auditeurs peut apporter au village. Les 
discussions ont abouti à l'identification des critères de bases pour la sélection des «candidats à l'innovation.»

\section{CRITERES POUR LA SELECTION DES AUDITRICES / AUDITEURS}

- être disponible à participer aux cours

- avoir la volonté d'apprendre

- être marié

- avoir au moins 18 ans

- avoir l'esprit de partage

- être quelqu'un qui peut donner des conseils

- tenir compte des éléments suivants : répartition par quartier, au plus un par concession

Initialement, le programme éducatif mis en œuvre au Sénégal ne comportait pas des classes pour hommes. Cependant le constat fait par TOSTAN au bout de quelques classes était que pour une meilleure mobilisation de la communauté les hommes devaient aussi être intégrés dans des classes. Ceci était particulièrement crucial dans le contexte du Burkina, où plusieurs enquêtes sur l'excision avaient montré le rôle prépondérant des hommes dans la prise de décision. C'est pourquoi, guidé par ces arguments, Mwangaza a décidé de prendre en compte le genre en intégrant les hommes dans la population cible de ce programme. Mandat a été donné aux membres du comité villageois de gestion, en collaboration avec les leaders des quartiers, d'établir la liste des participants en se basant sur les critères de sélection. Pour chaque village, il était attendu une liste de 30 auditrices et de 30 auditeurs.

A l'issue de la première sélection, 636 auditrices et 625 auditeurs ont été identifiés pour l'ensemble des 23 villages, soit un total de 1261 auditeurs recrutés sur les 1380 attendus, ce qui représente un taux de recrutement de $91 \%$.

\section{Phase de Mise en Oeuvre}

Il s'agit de toutes les actions de formation des facilitateurs communautaires, de renforcement des capacités des villages et de mise en œuvre d'activités au niveau communautaire.

\footnotetext{
Initiation des facilitateurs communautaires aux règles de transcription du Mooré Une des philosophies de MWANGAZA ACTION, dans le cadre de ses appuis aux populations, c'est de s'approprier « la langue et le langage » des communautés dans lesquelles elle intervient. Afin de se conformer à cette philosophie, une session de formation de six jours a été organisée au profit des facilitateurs communautaires. Cette session leur a permis de maîtriser les règles de transcription du mooré qui est la langue de travail dans la province du Zoundwéogo. Le contenu de la session a porté sur les points suivants :

\ présentation de l'alphabet national et celui du mooré

口 règles essentielles de transcription
} 
- présentation et lecture de nombres

- étude des voyelles orales brèves et longues

- étude des voyelles nasales brèves et longues.

Cette action est conforme à une des stratégies du programme qui est la post alphabétisation.

Formation des facilitateurs communautaires sur le contenu pédagogique

Deux séminaires de formation ont été organisés à l'intention des facilitateurs

communautaires. Une démarche itérative a été adoptée pour cela. Les séances ont alterné la théorie et la pratique.

Séminaire de formation sur les modules 1 et 2

Les facilitateurs communautaires recrutés pour la mise en œuvre étaient pour la plupart à leur première expérience de travail sur le terrain. Pour les outiller, un séminaire de formation sur le paquet pédagogique a été organisé lors de la première campagne du programme d'éducation à base communautaire. Ce premier séminaire de trois semaines a porté sur les thèmes suivants : l'éducation aux Droits Humains, le processus de résolution des problèmes, les techniques de conduite des séances, la technique d'utilisation de la boîte à images, la pédagogie des adultes, les techniques de tenue et d'animation des séances dans les classes.

Séminaire de formation sur les modules 3 et 4

Un deuxième séminaire de formation a été organisé durant la seconde campagne du programme. Ce séminaire de trois semaines était articulé autour des thèmes principaux suivants : Santé de la femme, les violences faites aux femmes, hygiène et prévention des maladies. D'autres thèmes comme la maternité à moindre risque, la politique nationale du Burkina en matière de vaccination, l'approche genre ont également été animés. Ce deuxième séminaire a contribué à améliorer les connaissances des facilitateurs communautaires sur :

- les différents appareils du corps humain ;

- l'anatomie et physiologie des organes de reproduction ;

- les pratiques traditionnelles néfastes à la santé de la femme ;

- les IST/VIH SIDA ;

- le calendrier vaccinal des enfants et des femmes ;

- Les actions à entreprendre pour une maternité à moindres risques ;

- l'approche genre ;

- l'organisation du système de santé au Burkina Faso.

Les points discutés durant le séminaire ont renforcé les connaissances des facilitateurs communautaires sur le contenu des modules 3 et 4 et ont permis de conduire efficacement les activités du programme éducatif dans les villages. Ces séminaires du type résidentiel ont permis à l'équipe d'encadrement de continuer à faire le « coaching » après les heures de travail, ce qui a énormément aidé les facilitateurs communautaires dans la maîtrise de la gestion de leurs futures classes. 
Outre l'équipe d'encadrement de MWANGAZA ACTION, les participants ont eu droit à des communications de personnes ressources spécialistes des questions des Droits Humains, du code des personnes et de la famille, de la santé et de genre. Ces communications ont renforcé les compétences techniques des facilitateurs communautaires.

Pour être opérationnel, chaque facilitateur communautaire a reçu pour son travail les matériels suivants :

口 Deux guides pédagogiques pour l'animation des modules 1 et 2 en français et en mooré

- Deux guides pédagogiques pour l'animation des modules 3 et 4 en français et en mooré

\Un lot de fiche bristol pour la préparation des séances

- Une boîte à image sur les droits humains

口 Un lot d'images pour les modules 2, 3 et 4

- Un lot de fiches et de cahiers pour le suivi des activités

a Un lit picot

¿ Une lampe tempête

a Un lot de matériels et fournitures de bureau

\section{Rencontre de prise de contact avec les membres des Comités Villageois de Gestion}

Entre la mise en place des comités villageois de gestion dans les villages et le démarrage des activités éducatives, il s'est écoulé deux mois. Afin de dynamiser les activités sur le terrain, une séance de travail s'est tenue entre facilitateurs communautaires et membres des Comités Villageois de Gestion. L'objectif de cette séance de travail était de faire la situation de l'état d'exécution des activités du programme. Les points qui ont constitué l'ordre du jour de ces séances de travail étaient :

a situation de la recherche de site pour la classe

口 état de l'aménagement de la classe

口 recrutement complémentaire de participants etc.

Dans chaque village une séance de travail a été tenue. Cela a permis le démarrage effectif de l'animation des séances avec les auditrices et les auditeurs.

\section{Formation des membres des Comités Villageois de Gestion}

Une session de deux (2) jours a regroupé les membres des comités villageois de gestion. Le thème de la session était centré sur les rôles et responsabilités des membres du comité villageois de gestion. Durant cette session, les connaissances des participants ont été renforcées sur la technique de gestion des réunions.

\section{Equipement des villages}

Afin d'assurer un minimum de confort pour le travail dans les villages et une animation acceptable des séances, un équipement minimal était nécessaire. Le programme a doté chaque classe de matériels et fournitures suivants :

口 Six bancs 
- Une corde à linge pour accrocher les images

- Un lot de pinces à linge

- Un cahier et un crayon pour chaque participant.

\section{Détermination des modalités pratiques de démarrage des séances dans les villages}

Une séance de travail a eu lieu entre chaque facilitateur communautaire et les auditrices et les auditeurs. Cette première rencontre de présentation et de connaissance mutuelle a servi de cadre pour la négociation avec chaque groupe d'auditrices et d'auditeurs, des modalités pratiques de démarrage du programme éducatif. Ces négociations ont porté sur les jours, les horaires des classes et la date de début effectif des séances pour chaque sexe. La séance de travail a été l'occasion de faire un rappel des grandes lignes du programme.

\section{Animation des modules dans les villages}

Les auditrices, les auditeurs et les facilitateurs communautaires, conformément aux modalités arrêtées lors de la séance de travail avec les participants, ont mis en œuvre le programme éducatif. Les séances des modules Droits Humains et processus de résolution des problèmes sont animées au profit des participants tous les deux jours. Des activités de promotion des Droits Humains et de résolution des problèmes sont entreprises dans la classe et dans le village par les participants.

Les méthodes participatives, où l'apprenant est l'acteur principal des activités de formation sont privilégiées. Les techniques actives suivantes sont utilisées durant l'animation des séances : la danse, le chant, le jeu de rôle, le poème, le travail en petits groupes. Les classes sont aménagées en forme de U. Cet aménagement permet à ceux qui prennent part aux séances de se voir sans être obligés de se retourner lorsque l'un d'eux prend la parole.

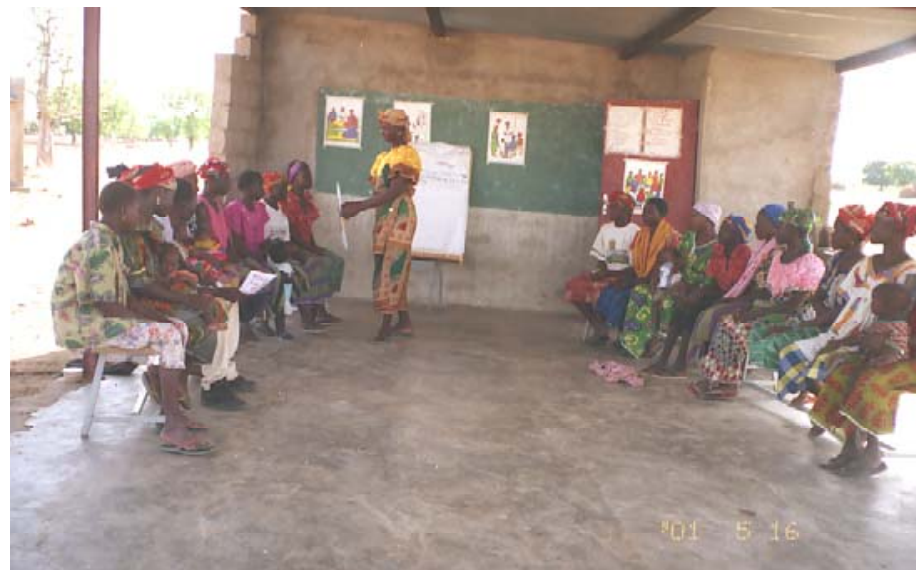

Séance éducative sur les Droits Humains avec les auditrices du village de Noghin (Département de Binde)

Le facilitateur communautaire est assis au même niveau que les participants durant toute la durée des séances. Ceci rompt avec les habitudes du système éducatif formel où le facilitateur est debout (ascendant sur les autres) durant ses prestations.

L'animation de la séance du jour commence par la présentation du flip chart comportant le numéro, le titre et les objectifs de la séance. Le facilitateur suit ensuite le fil conducteur noté dans les fiches bristol pour l'animation de la séance. 
L'animation des séances prend toujours fin après l'évaluation, où participants et facilitateurs passent en revu les objectifs du jour et les résultats atteints. Après l'appel, les participants rentrent chez eux.

Le programme éducatif comprend 63 séances. Ces séances sont animées en deux campagnes. La première va de février en mai 2001. Elle concerne les modules éducation aux Droits Humains et processus de résolution des problèmes. La seconde s'est déroulée de décembre 2001 en mars 2002 pour les modules hygiène et prévention des maladies et santé de la femme.

\section{Animation de séance publique}

Concomitamment aux séances dans les classes, les facilitateurs communautaires, à la demande des auditeurs animent des séances publiques. Elles consistent à organiser des séances d'animation hors de la classe, pour un grand public, pour les personnes qui ne participent pas aux séances dans les classes. Ce sont les auditrices et auditeurs euxmêmes qui sont à la base de l'organisation et de l'animation des séances.

Deux situations ont été enregistrées, il y a d'abord les séances publiques dans les villages du programme et les séances publiques dans les villages non couverts par le programme. Les séances qui sont animées dans les

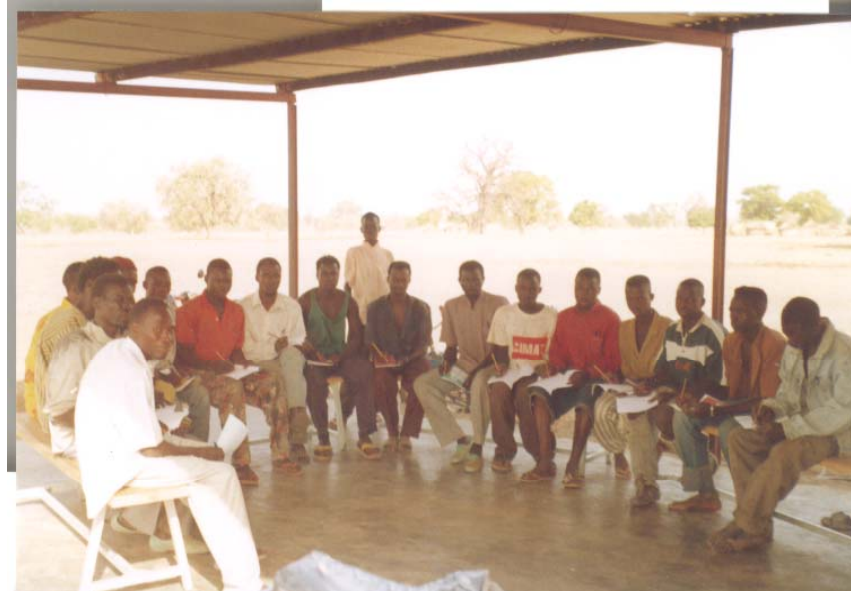

Séance éducative sur la résolution des problèmes avec les auditeurs du village de Nacombgo (Département Béré) villages sont :

a l'excision,

- la grossesse et la planification familiale,

- le droit à la santé,

- le droit à un environnement sain.

a la sexualité

a les IST / VIH/ SIDA.

Au niveau des villages du programme, l'objectif recherché est d'informer toute la communauté afin qu'il y ait un soutien aux activités que les auditrices et auditeurs veulent mettre en œuvre. Concernant les animations dans les villages qui ne sont pas couvert par le programme, il s'agit aussi de les informer afin qu'ils adhèrent aux différentes stratégies que les 23 villages développent. La population a fortement apprécié l'initiative de l'animation de ces séances publiques. 


\section{Communication sociale}

Sous la forme de grandes rencontres, des échanges ont lieu entre les membres d'un même village et entre populations de plusieurs villages relevant d'une même aire de santé.

1. Réunions villageoises

Une réunion villageoise a été organisée dans chacun de 23 villages. Les auditrices, les auditeurs et les membres des Comités Villageois de Gestion sont les principaux acteurs de ces rencontres. Ces acteurs ont fait à la communauté le point de l'exécution du programme dans le village. Les acquis sont présentés, les forces et les faiblesses discutées, des solutions pour la résolution des problèmes sont proposées. Ce bilan est fait à l'aide de chants, de danses, de sketches et de discours. Un total de 6500 personnes a pris part à ces rencontres

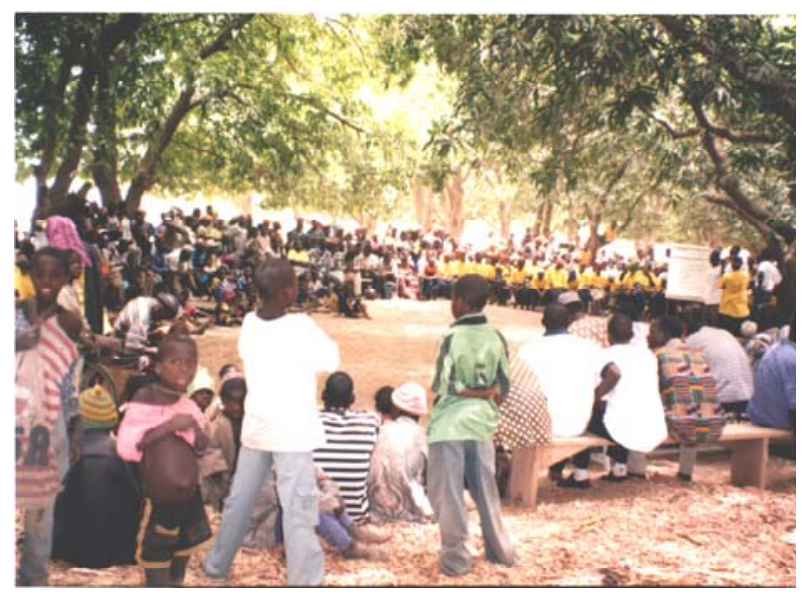

Réunion inter-village regroupant les délégations des 5 villages à Sondré (Département Béré) villageoises.

Ces réunions ont été un tremplin pour les rencontres inter-villages. Chaque village a déterminé et présenté sa communication à la rencontre inter-villages.

\section{Rencontres inter-villages.}

Les villages d'une même aire de santé (environ 8 villages par aire de santé) se sont retrouvés pour faire le point du déroulement des activités du programme à la fin de chaque campagne.

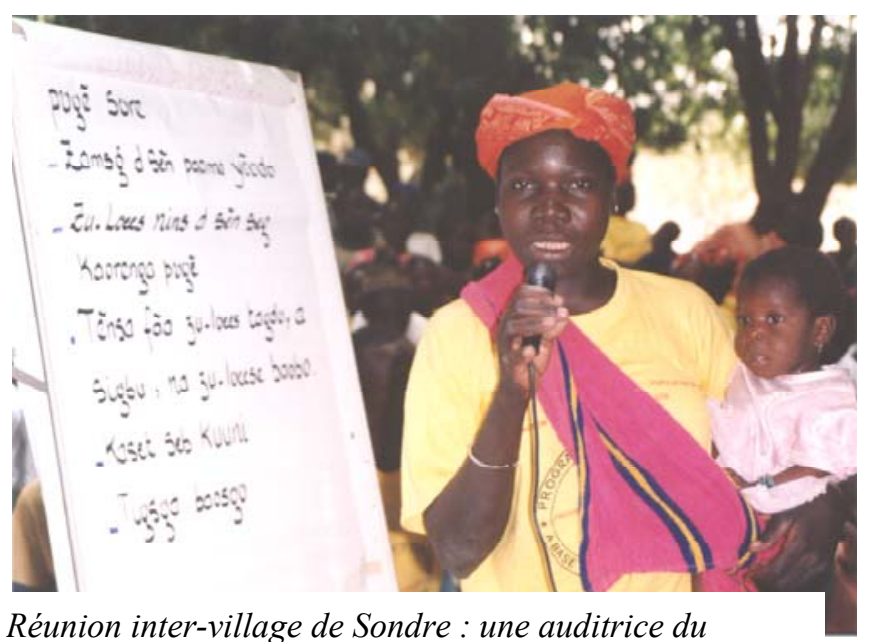

village de Signoghin présentant les problèmes auxquels son village veut apporter des solutions.
Au cours de ces rencontres, qui ont duré une journée chacune, les délégations villageoises ont échangé devant les leaders de la communauté, les partenaires au développement, l'administration, sur les réalisations, les acquis, les forces et les faiblesses du programme.

Les participants ont également discuté et proposé des solutions aux problèmes qui sont communs à l'ensemble des villages. Des actions communes ont été programmées. Six rencontres inter-villages ont été 
animées. Les trois premières à la fin des deux premiers modules et les trois dernières à la fin des modules 3 et 4 .

Le première rencontre, tenue à la fin de la première campagne, a recommandé la nécessité pour la communauté de participer aux activités du programme d'éducation pour un meilleur renforcement de leurs capacités. La deuxième a mis l'accent sur l'impérieuse nécessité de lutter contre les violences faites aux femmes avec un accent particulier sur la lutte contre la pratique de l'excision. Environ 8000 personnes ont participé à ces rencontres.

\section{Phase de Suivi et d'Evaluation}

\section{Suivi des activités}

Le suivi des activités et la supervision des facilitateurs relèvent de la tâche des superviseurs. Chaque superviseur avait sous sa responsabilité un maximum de 8 facilitateurs communautaires. Lors des rencontres mensuelles de chaque zone de supervision, chaque facilitateur fait le compte rendu des ses activités et présente son programme mensuel. Sur la base ces programmes, le superviseur effectue ses visites dans les villages pour appuyer la mise en ouvre des activités de chaque village.

Au niveau des villages, le suivi des activités est fait par les membres des comités villageois de gestion. Le contact est permanent entre superviseur et Comités Villageois de Gestion. Des rencontres mensuelles de l'équipe d'encadrement ont lieu. Le point de la mise en œuvre des activités est fait lors de ces rencontres. Les solutions aux difficultés rencontrées sont proposées.

\section{Evaluation du programme}

L'évaluation formative est faite de façon permanente. Ainsi durant l'animation des séances et à la fin de chaque module des activités d'évaluation ont été menées.

\section{DYNAMIQUE COMMUNAUTAIRE}

Dans les classes, nous avons constaté avec intérêt, des réactions positives dans la plupart des villages, suite à l'animation des séances du programme éducatif. Ces réactions étaient spontanées et diverses. Elles consistent en l'identification et la mise en œuvre d'une action résultante des discussions qui se sont déroulées dans les classes. Ces actions ont commencé au début timidement dans trois villages puis ont fait tâche d'huile pour atteindre l'ensemble des 23 villages. Pour aboutir à ce résultat, un système de dissémination des actions villageoises a été mis en œuvre.

A cet effet, l'expérience de chaque village est lue et commentée dans toutes les classes, lors de l'animation des séances. Par cet acte, l'expérience présentée dans la classe donnait de nouvelles idées aux participants de la classe et constituait une source de motivation et d'inspiration pour les participants. Il s'agit d'amener ces participants à entreprendre des actions afin que l'expérience de leurs villages soit présentée aussi aux autres villages. Les 
premières actions entreprises sont : opération de nettoyage de lieux publics (dispensaires, écoles) ; dénonciation des cas de violences et de discrimination (femmes battues, enfants n'allant pas à l'école).

Par la suite d'autres actions aussi importantes les unes que les autres ont été entreprises. Ces actions peuvent être classées en trois catégories :

Nettoyage et embellissement des lieux publics

- Fabrication de plus de 89 foyers améliorés

- Nettoyage de point d'eau dans tous les 23 villages

- Nettoyage des marchés

- Nettoyage des cours des chefs et des préfectures

- Institution de journées de salubrité dans tous les 23 villages

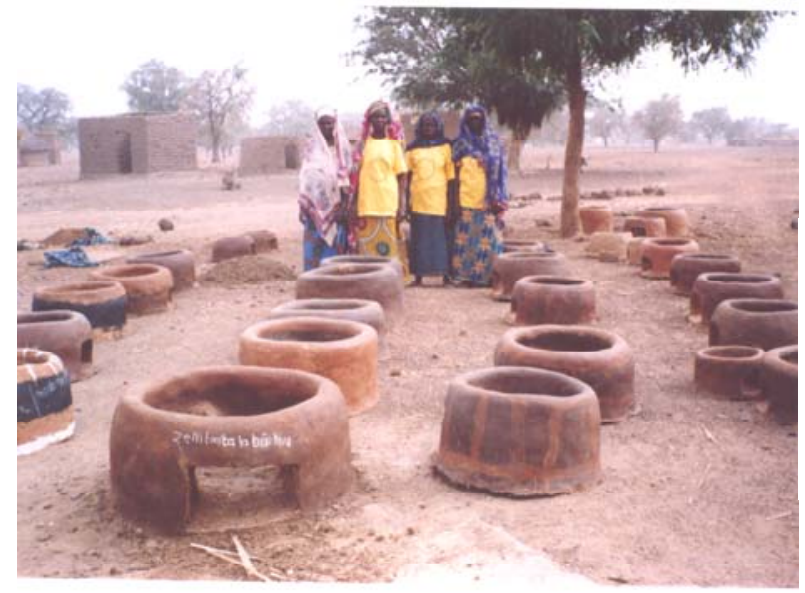

Des auditrices $d u$ village de Kondrin devant les foyers améliorés qu'elles ont fabriqués.

Promotion de la santé

- Nettoyage des centres de santé et de promotion sociale (dispensaires et maternités)

- Dynamisation de 10 postes de santé primaire

- Présentations de pièces de théâtres sur la santé de la femme

- 27 séances publiques animées dans 11 villages sur les thèmes suivants : 1'hygiène, l'excision, la sexualité, l'espacement des naissances, les IST / VIH / SIDA, la grossesse, la vaccination

- 23 groupes de promotion de la santé mis en place dans les 23 villages.

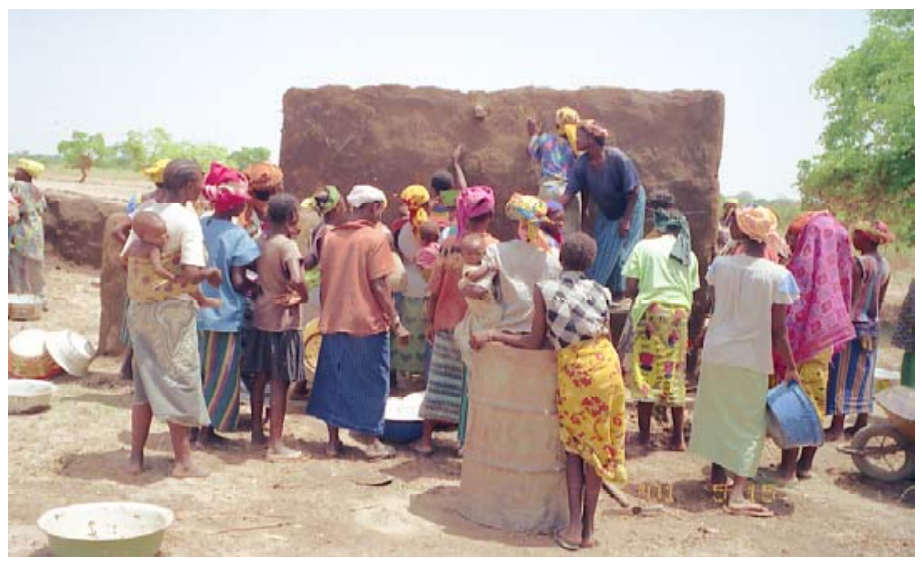

Des femmes du village de Yorgho en train de renforcer les murs de leur poste de santé primaire avec un enduit de banco. 
Promotion des Droits Humains

- Dénonciation d'un cas de mariage forcé

a Discussion sur les violences faites aux femmes dans tous les 23 villages

- 69 personnes ont participé à un atelier de résolution des violences faites aux femmes

- Constatation d'un cas de discrimination par rapport à l'éducation des enfants

- Etablissement de 684 actes de naissances

a Etablissement de 78 livrets de famille

- Etablissement de 693 cartes d'identité

- Célébration d'un mariage collectif pour 43 couples

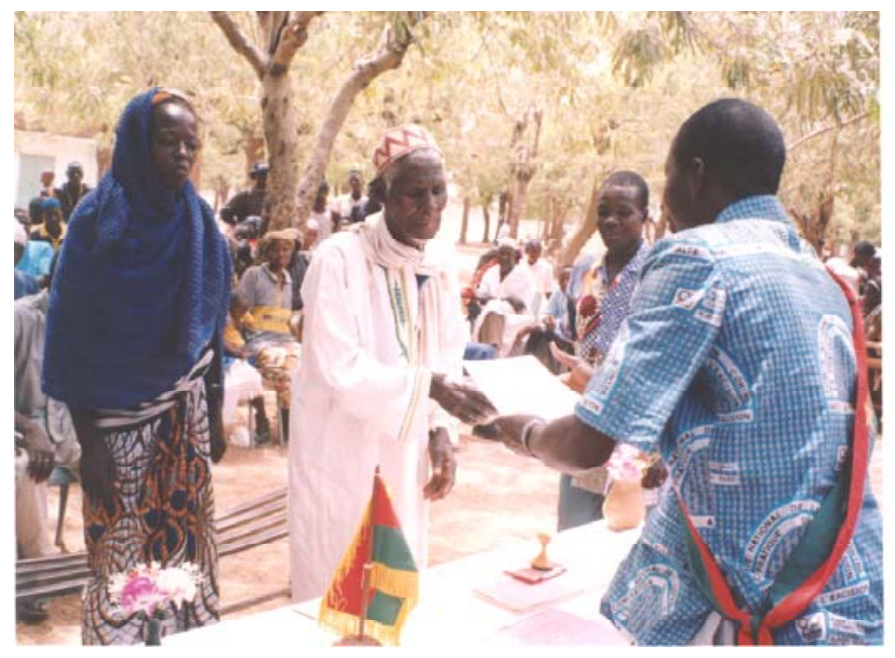

Cérémonie publique de mariage à Béré : une auditrice et son mari reçoivent des mains $d u$ Préfet le livret de famille suite à la régularisation de leur situation matrimoniale.

Ces actions constituent la preuve du dynamisme de la population mais aussi de l'assimilation du contenu des séances par les participants et leurs conjoints.

\section{RESULTATS DE L'INTERVENTION}

Les résultats majeurs obtenus par le programme d'éducation à base communautaire dans les villages sont de plusieurs ordres :

\section{Amélioration des connaissances de la population}

Les différentes évaluations faites pendant et après l'animation des séances du programme éducatif montrent une amélioration des connaissances des populations sur le contenu des quatre modules développés dans les villages. Les connaissances sont beaucoup plus accrues au niveau des auditrices, des auditeurs, des conjoints et des amis

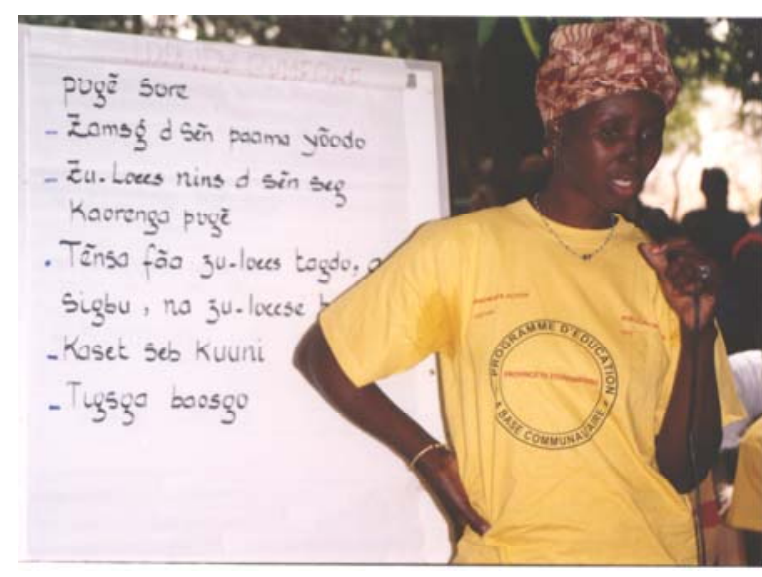

Une auditrice de Nacombgo présente les acquis $d u$ programme dans son village, lors d'une réunion inter-village à Sondré 
avec lesquels les informations reçues en classe ont été partagées. L'effectif total des personnes formées est de 1674 : 907 femmes et 767 hommes ; avec une moyenne de 73 par village.

\section{Actions communautaires dans les villages}

Un autre aspect des résultats du programme c'est le développement d'actions au sein de la communauté par les participants, en collaboration avec les autres habitants des villages. Les actions entreprises concernent l'amélioration de la santé de la population, l'amélioration du cadre de vie, la promotion des droits en général et ceux des femmes et des enfants en particulier. Toutes ces actions sont la résultante de l'amélioration des connaissances des populations.

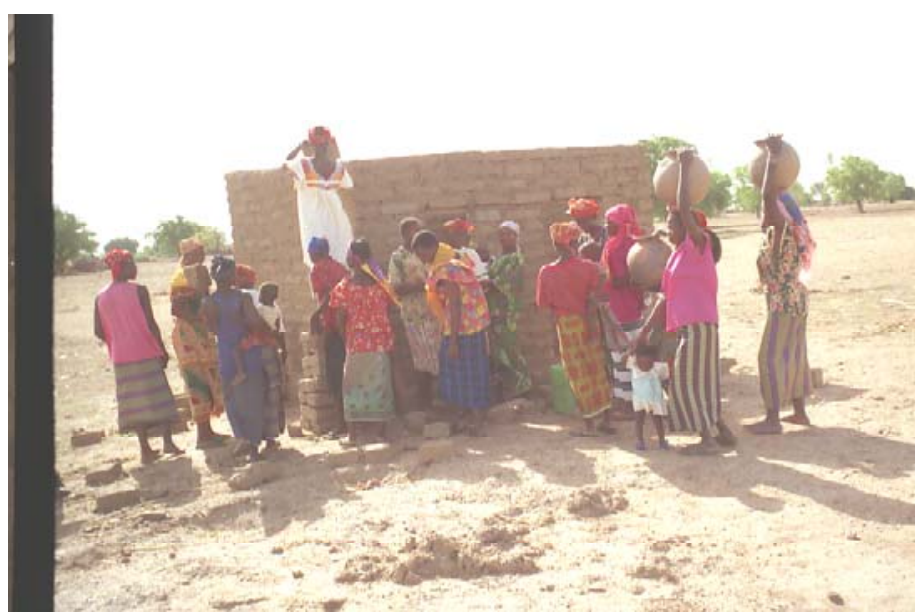

Des femmes de Doué participent à la construction du poste de santé primaire $d u$ village.

3. Communication sociale sur les violences à l'égard des femmes

Les discussions sur les violences à l'égard des femmes et des filles comme l'excision, les mariages précoces et forcés, les accouchements rapprochés qui autrefois étaient des sujets tabous ne le sont plus. Les femmes en discutent ouvertement avec les hommes et les leaders de la communauté. Cela s'est concrétisé par l'organisation d'un atelier communautaire sur les violences qui sont faites aux femmes. Soixante neuf (69) personnes venant des 23 villages se sont retrouvées pour échanger sur les problèmes qui leur sont communs. Les communautés à travers leurs représentants (femmes et hommes) ont procédé au diagnostic de la situation de la santé sexuelle et reproductive. Les outils et techniques tels que, la matrice de prise de décision, le pourquoi-pourquoi ?, le diagramme de Gantt, ont été utilisés. A

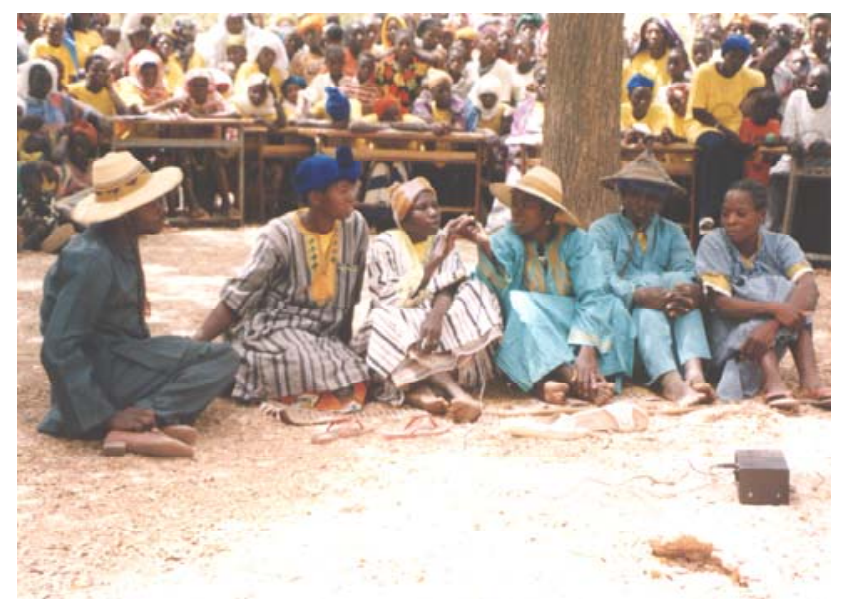

Un théâtre sur le VIH/SIDA présenté par les auditrices de Siguinvousse lors d'une réunion inter-village regroupant 11 villages à Béré 
les villageois ont identifié les problèmes de santé, les ont analysés pour rechercher les causes afin de les résoudre.

Un plan d'action commun à l'ensemble des 23 villages a été élaboré dans le cadre de la recherche de solutions aux problèmes suivants :

1. Les naissances rapprochées

2. La pratique de l'excision

3. La pratique des mariages forcés et précoces

4. La propagation des IST/VIH/SIDA

5. Les violences faites aux femmes

6. L'insuffisance de l'alphabétisation.

Le programme d'éducation à base communautaire a été un facteur de changement au sein des communautés des 23 villages. Ainsi les avis ci-dessous de la population sont révélateurs des changements qu'elle a constatés :

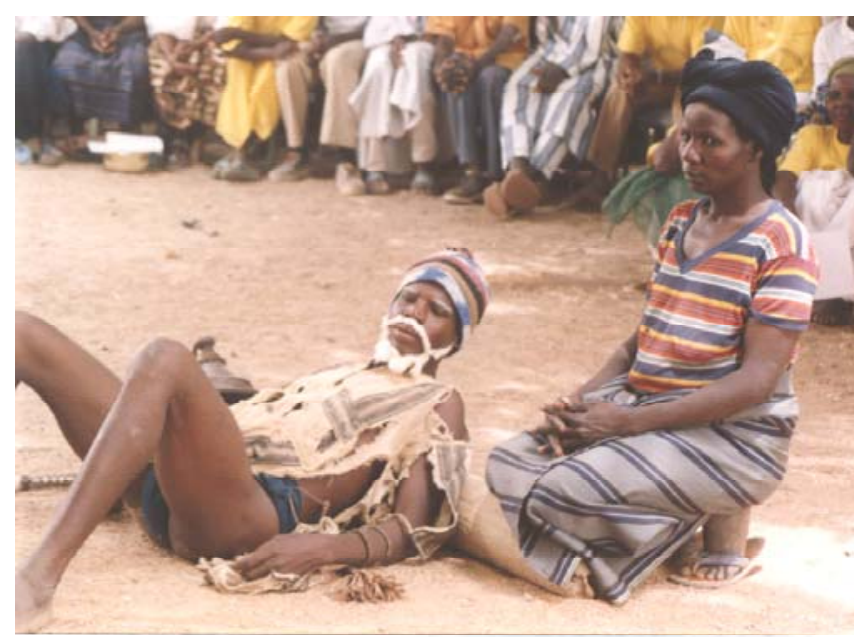

Des auditeurs et auditrices de Bindé présentent un théâtre sur les méfaits de la pratique de l'excision

- "Vos enseignements nous ont permis de nous rapprocher de nos femmes. Il n'y a plus de complexe dans nos rapports, c'est pour cela que les hommes exigent la reprise de certaines séances pour leurs conjointes » (Auditeur, village de Signoghin, Département de Béré)

Les auditeurs connaissent désormais mieux les problèmes de santé de leurs femmes

- "Vos enseignements sur la santé nous ont permis de mieux connaître notre organisme et les méthodes contraceptives » (Auditrice, village de Sondré, Département de Béré)

- "Vos enseignements ont libéré la parole des femmes. Maintenant, elles peuvent parler d'elles-mêmes » (Animateur, FARD/ONG de Développement, Département de Bindé) 
- "Il y a eu une utilisation accrue des services de santé :consultation prénatale, soins, accouchements...» (Infirmier chef de poste, Centre de Santé et de Promotion Sociale de Bindé, Département de Bindé)

- "Plus de considération pour les femmes » de plus en plus la femme se voit consulter pour les prises de décision, la résolution de certaines questions jadis réservées aux hommes. (Auditeur, village de Béré, Département de Béré)

- "Implication des autorités coutumières dans la lutte contre l'excision et les naissances rapprochées ». (Auditrice, village de Kondrin, Département de Béré)

Participation des responsables coutumiers à la réunion inter-village à Sondré

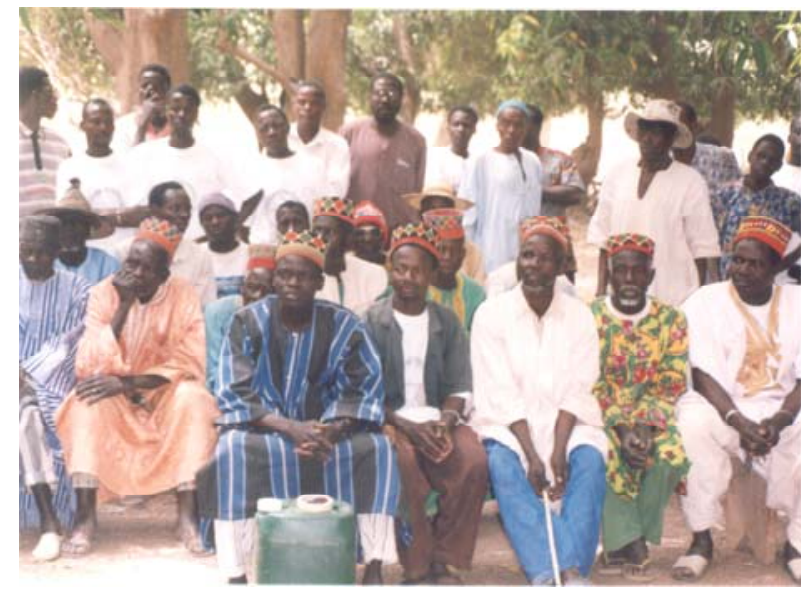

\section{ENGAGEMENT DES 23 VILLAGES DEVANT 5000 PERSONNES}

Prendre un engagement collectif et public pour faire ou ne pas faire quelque chose est un acte d'une grande importance dans le processus de changement de comportement. Cet acte collectif a été posé par les populations de Béré et de Bindé le 3 mai 2003. Environ 5000 personnes ont pris part à la cérémonie de déclaration d'abandon de la pratique de l'excision.

On peut situer l'importance de cet acte à deux niveaux. D'abord au niveau de la communauté ayant pris l'engagement d'abandonner la pratique de l'excision. Pour la population de Béré et de Bindé, c'est un tournant de la vie part rapport à la pratique de l'excision et à la promotion de la santé et droits de la femme et de la petite fille. Le fait de prendre publiquement position est un acte courageux mais aussi responsable. Ils ont pris le temps de comprendre et en toute connaissance de cause ils ont décidé
Placé sous le Haut Patronage du Ministère de l'Action Sociale et de la Solidarité Nationale, la cérémonie de déclaration publique d'abandon de la pratique de l'excision a connu la participation des populations des 23 villages y compris ceux des villages environnants, des autorités coutumières et administratives, des services techniques, des associations et ONG, des Parlementaires, des Partenaires au développement comme UNICEF, UNFPA, ZONTA Club. La presse était également présente à travers les médias nationaux et internationaux. Les partenaires ayant soutenu MWANGAZA ACTION dans la mise en oeuvre du programme d'éducation à base communautaire ont massivement honoré de leur présence à la cérémonie de déclaration publique de Béré, notamment: TOSTAN, POPULATION COUNCIL, GTZ, CNLPE, UERD. 
d'abandonner la pratique de l'excision. On peut dire qu'en officialisant cette décision, la population de Béré et de Bindé a posé un acte de grande portée. Une action collective.

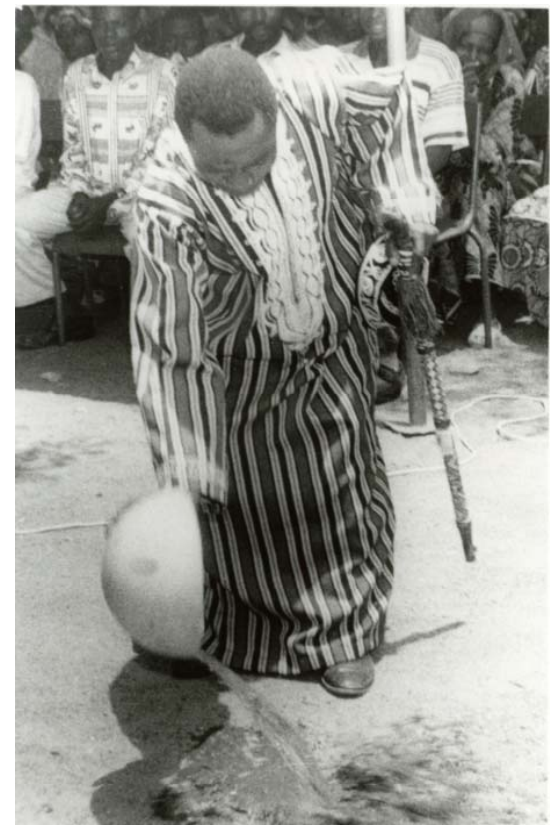

Pour les autres communautés Burkinabé, avec l'appui des media qui ont relayé l'événement aux quatre coins du Pays, l'acte posé par les gens de Béré et de Bindé donne et indique le chemin à suivre pour l'éradication de la pratique de l'excision. Béré et Bindé seront des modèles dans la démarche communautaire pour l'abandon de l'excision.

Par ce geste rituel, cet homme a été mandaté par les chefs coutumiers à valider, au nom des ancêtres, l'engagement pris par les femmes à Béré d'abandonner la pratique de l'excision
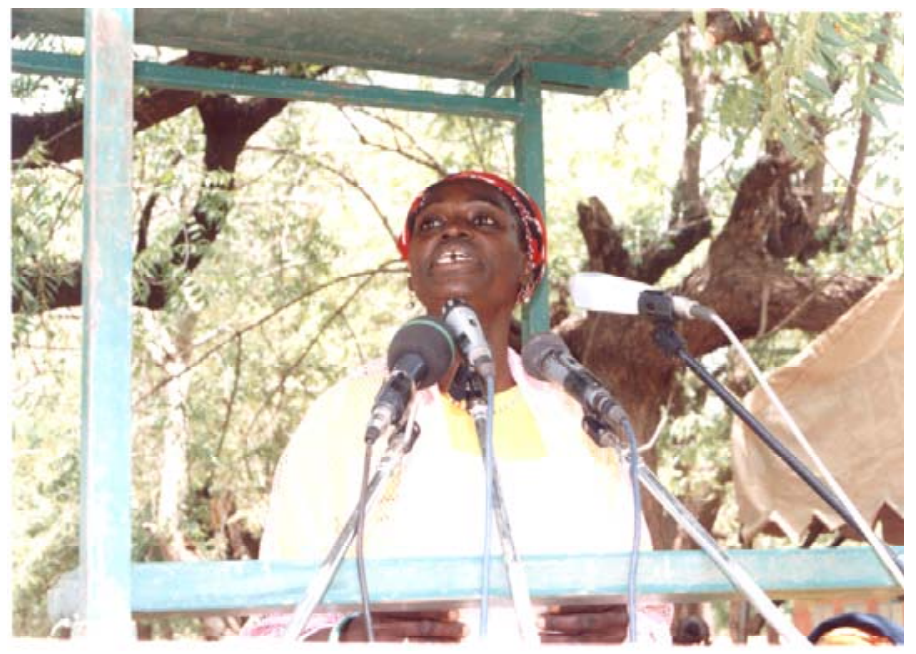

Du haut de cette tribune, dressée pour la circonstance, cette femme prononce au nom de toutes les communautés la déclaration publique d'abandon de l'excision lors de la cérémonie de Béré. 


\section{DECLARATION PUBLIQUE D'ABANDON DE LA PRATIQUE DE L'EXCISION Béré le 3 Mai 2003}

Les populations de 23 villages des départements de Béré et de Bindé par ma voix voudraient porter à votre connaissance notre décision de mettre un terme aujourd'hui à la pratique de l'excision dans notre communauté.

Au Burkina Faso, minimes sont les communautés qui ne pratiquaient pas l'excision. La population de Béré et de Bindé fait partie de ceux qui la pratiquaient. Nous pratiquions l'excision :

- parce que nous pensions que c'était une bonne chose pour nos enfants,

- parce que nous étions analphabètes,

- parce que nous ignorions les conséquences d'une telle pratique sur la santé,

- parce que nous ignorions même le fonctionnement de notre corps et enfin

- parce que nous ignorions que tout être humain à des droits, comme celui de disposer de son corps.

Chers invités, depuis deux ans, la situation dans nos communautés a quelque peu changé. Hier dans les ténèbres, aujourd'hui nous recevons une sorte de lumière à travers le programme éducatif de Mwangaza. Grâce donc à ce programme nous voyons de plus en plus clair certains maux dont nous souffrions hier.

Nous savons désormais que, quoique faisant partie de nos valeurs traditionnelles, l'excision est une pratique dangereuse pour la santé de la femme et de la fille.

Nous savons désormais aussi qu'il existe des droits reconnus à tout être humain de disposer de son corps dans son intégralité.

Nous savons enfin que nous devons entreprendre des actions pour la promotion de la santé et les droits de la personne humaine dans notre communauté.

A la lumière de tous ces constats, et en toute connaissance de cause, nous voudrions vous prendre à témoin, vous nos illustres hôtes à cette cérémonie, de notre engagement suivant :

Nous populations de 23 villages des départements de Béré et de Bindé, nous engageons solennellement à entreprendre des actions pour la promotion de la santé et pour le respect des droits humains dans notre communauté. Par conséquent, en cette date du 3 mai 2003, nous décidons de mettre fin à la pratique de l'excision dans tous nos villages.

Je vous remercie 
Peut-on dire que la déclaration publique d'abandon de la pratique de l'excision faite à Béré est l'aboutissement d'une action collective ? Quels sont les facteurs qui sont à la base de cette action collective?

\section{Les éléments à la base du changement social dans les communautés de Béré et de Bindé}

La démarche, qui a été suivie pour accompagner les communautés des départements de Béré et de Bindé dans la déclaration publique pour l'abandon de l'excision, comprend les étapes suivantes :

\section{Atelier de résolution des problèmes}

On dit souvent que l'union fait la force. Dans le cadre du programme d'éducation à base communautaire, les connaissances acquises par les populations des 23 villages les ont amenées à engager une lutte contre certains problèmes qui leur sont communs et qui minent leur vie. Ces problèmes sont : les violences à l'égard des femmes, le mariage forcés et précoces, l'insuffisance de programme d'alphabétisation, l'insuffisance de services d'espacement de naissance et de planification familiale, la propagation du VIH/SIDA, la pratique de l'excision.

Des représentants des 23 villages, après avoir hiérarchisé les problèmes, ont procédé à l'analyse et à la planification des solutions. Cet atelier a été le premier engagement des populations pour l'éradication de certains maux comme l'excision.

Les participants à cet atelier sont des acteurs du changement, composés d'auditrices et d'auditeurs, de responsables coutumiers et religieux, ces personnes ont tracé la voie à suivre pour un changement au sein de la communauté en ce qui concerne la question des violences à l'égard des femmes dont l'excision.

\section{Visite d'échanges avec la population de Kolda Sénégal}

Dans le cycle de l'apprentissage par l'expérience, «l'apprenant apprend en se servant de ses propres expériences ». Dès lors, l'expérience constitue la première étape du cycle.

Parmi les techniques utilisées pour faire apprendre ou maîtriser une compétence, on peut citer le voyage d'échange d'expérience. A cet effet, une délégation a séjourné au Sénégal afin de prendre part à la cérémonie de déclaration publique d'abandon de l'excision qui s'est déroulée à Karcia ( $30 \mathrm{~km}$ de Kolda) le 5 juin 2002. Cette déclaration publique a regroupé un total de 300 villages et a vu la participation de plusieurs organisations de lutte contre la pratique de l'excision, dont le réseau des parlementaires sénégalais en population.

Durant ce séjour, les membres de la délégation Burkinabè, composés de trois personnes venant de la zone d'intervention et du chargé de programme de MWANGAZA ACTION, ont échangé avec la population sénégalaise sur le déroulement de leur programme 
éducatif, les relations entre villages et tout ce qui a contribué à la prise de décision en faveur de la santé de la femme et de ses droits.

Cet échange a été un catalyseur et a contribué à renforcer la conviction de la délégation Burkinabè sur l'impérieuse nécessité de continuer à promouvoir la santé et le droit des femmes et des filles.

\section{Dialogue intercommunautaire}

Les auditrices, les auditeurs et les membres de la délégation (surtout les villageois) ayant séjourné au Sénégal ont entrepris de parler avec toutes les couches de la communauté (responsables coutumiers, responsables administratifs, responsables religieux, population en général ) pour d'une part partager l'expérience vécue lors de leur séjour au Sénégal et d'autre part rappeler les apprentissages et autres acquisitions faites durant le déroulement du programme d'éducation à base communautaire sur la santé et le droit de la femme.

Des réunions villageoises et inter villages ont été le cadre de ce dialogue inter communautaire.

Ces rencontres villageoises et inter villages ont été pour toute la population, avec l'appui des notabilités, l'occasion de prendre des engagements pour l'abandon de la pratique de l'excision dans l'ensemble des 23 villages. Ces engagements ont été pris au niveau de chaque village ensuite au niveau de deux réunions inter villages tenues à Béré et Bindé.

L'engagement des communautés pour l'abandon de la pratique de l'excision dans les deux chefs lieux de départements s'est fait devant les responsables de l'Administration, des Services techniques et des Associations et $\mathrm{ONG}$ intervenants à Béré et à Bindé.

Le succès de ces activités témoignent de l'engagement irréversible de la population pour la promotion de la santé et droits de la femme avec une lutte particulière pour l'abandon de la pratique de l'excision.

\section{Atelier de planification des activités de la déclaration publique d'abandon de la pratique de l'excision}

Un atelier a été organisé, dont l'objet a été de mener une réflexion sur comment officialiser la décision de la population par rapport à l'abandon de la pratique de l'excision. L'atelier a regroupé les représentants des 23 villages. Etaient également présents à titre d'observateurs, les responsables de l'Administration, des Services techniques et des Associations et ONG intervenants à Béré et à Bindé

Les objectifs de l'atelier étaient : 1) Echanger entre représentants des villages sur les aspects pratiques de l'engagement de la communauté pour l'abandon de la pratique de l'excision. 2) Identifier et planifier les activités rentrant dans le cadre de cet engagement. 
Outres les aspects organisationnels, les discussions se sont focalisées sur la signification et l'importance de la parole donnée dans les communautés de Béré et de Bindé. Les procédures pour un engagement collectif et les mécanismes de suivi des décisions prises ont également été abordés.

Entre autres décisions prises à cet atelier, il a été retenu qu'une femme prendra la parole pour présenter à l'assistance la décision de la collectivité de Béré et de Bindé. Elle sera suivie par un représentant des responsables coutumiers qui posera un geste symbolique d'engagement et de soutien à la décision de la population de mettre un terme à la pratique de l'excision dans les limites territoriales des deux départements.

Que retenir d'une telle action collective?

\section{Détermination des femmes}

Dans la lutte contre les violences et pour la promotion de leur droits reproductifs, les femmes des deux départements se sont fortement mobilisées depuis le début du programme dans les 23 villages. En tant que « victimes », les femmes se sont résolument engagées dans la lutte contre les violences dont elles sont l'objet. Tout le long de la mise en œuvre du programme, elles n'ont cessé d'être au devant de la scène pour exprimer leur détermination à mettre fin à

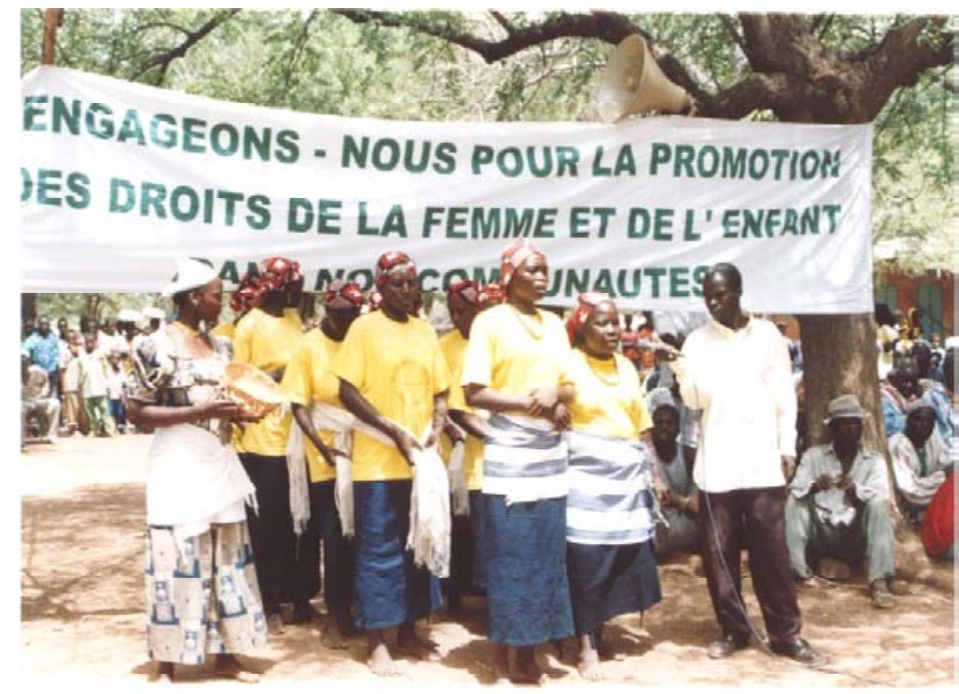

Ce groupe de femmes entonne une chanson appelant à l'abandon de la pratique de l'excision dans leur communauté pendant la déclaration publique de Béré.

l'excision et à promouvoir le

bien-être de leurs progénitures filles. Les femmes des deux localités n'ont ménagé aucun effort pour cette lutte. Elles ont bravé les intempéries, la chaleur, et ont parcouru de longues distances à vélos pour participer à des rencontres communautaires pour prendre la parole afin que la spécificité, les besoins de la femme soient pris en compte dans les instances de décision au niveau communautaire. 


\section{Engagement des hommes et des notables}

« L'homme n'est rien sans la femme ». Les hommes se sont vraiment engagés au coté des femmes pour l'aboutissement de la lutte pour le bien être de la femme. Les hommes en général, et les notables (leaders) en particuliers, ont fait preuve de clairvoyance dans la lutte contre l'excision. Ils ont toujours répondu à l'appel pour des actions en faveur de la femme. Les leaders

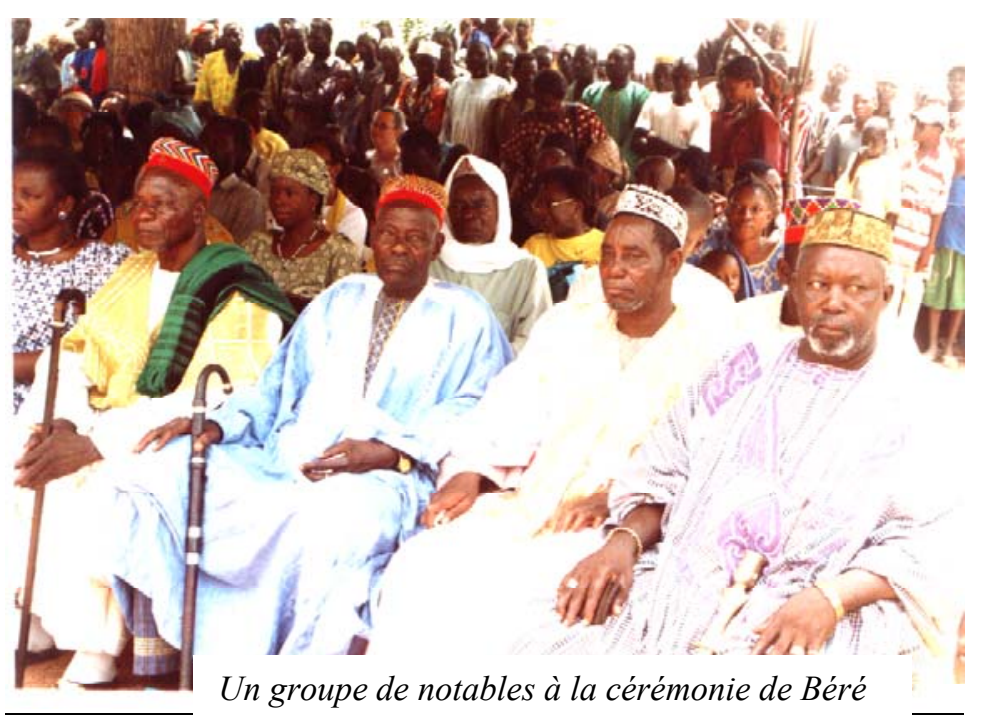
(coutumiers et religieux) communautaires ont favorisé l'union sacrée autour de la question concernant la santé et le droit de la femme.

\section{Mobilisation des médias}

La mobilisation des médias dans le cadre de l'organisation de la cérémonie publique d'abandon de la pratique de l'excision de Béré a été à la hauteur des attentes qui étaient de donner à cet événement une plus grande visibilité. Les média ont effectivement joué un rôle très important en ce sens que l'événement a été relayé aux quatre coins du Burkina Faso et même au-delà des frontières burbinabès grâce à la couverture médiatique de l'Agence panafricaine de presse (PANA), la BBC Africa et la Voice of America dans ses éditions africaines.

Plus concrètement, 10 articles de presse ont été publiés par la presse nationale et internationale avant, pendant et après la cérémonie de la déclaration publique de Béré. La Télévision nationale burbinabé (TNB) a fait deux fois écho de l'événement, la veille et le jour de la cérémonie, dans ses plus regardées éditions télévisées de 20h30. La Radio nationale burkinabé (RNB) et la Radio rurale ont également annoncé l'événement dans leurs journaux parlés. Le plus important c'est qu'au niveau du Burkina, l'information a été reprise dans les principales langues que sont le Mooré, le Dioula, le Fulfuldé et le Gulmancema.

Cette forte implication des médias s'inscrit dans le cadre du programme TOSTAN qui fait des médias l'un des piliers de sa stratégie de mobilisation sociale. Cependant, la particularité de la démarche adoptée par MWANGAZA et POPULATION COUNCIL 
réside dans la mise en œuvre et l'adaptation d'un plan média au paysage médiatique burkinabé. Exécuté en trois phases, ce plan cherchait d'une part à briefer les journalistes sur le caractère sensible du thème lié à l'excision dans la société burkinabé, avant de gagner leur adhésion. D'autre part, compte tenu du budget limité, il était important de négocier avec les médias en jouant sur la corde sociale de l'événement. En effet, dépourvus de publicités et de moyens de fonctionnement, les médias burkinabé n'hésitent pas à demander une forte somme pour assurer la couverture d'un événement.

A travers le plan média, tous les responsables des journaux, radios et télévision ont été directement ciblés par des visites de courtoisie pour être sensibilisés sur l'intérêt de l'événement et la nécessité de sa couverture médiatique. Les rencontres avec les responsables ont été fructueuses, et cela grâce en partie au soutien de Pop'Média Burkina, un large réseau des journalistes en population, rassemblant tous les responsables des médias burkinabé. Après l'accord de principe des responsables des médias, des visites de prise de contact et d'information ont été organisées, visant les rédactions des différents organes de la presse ciblés. Tous ces contacts ont débouché sur un déjeuner de presse, regroupant 18 journalistes, organisé le 29 avril 2003, trois jours avant la cérémonie. Ce déjeuner a été non seulement l'occasion de bien informer les journalistes par un parterre d'experts en santé de la reproduction avant la couverture de la cérémonie, mais il s'est beaucoup plus agi d'installer un climat de confiance entre les journalistes et tous les autres acteurs.

Cette stratégie de communication s'est révélée très efficace car dans leur totalité, tous les organes de presse invités ont répondu présents à l'appel. En plus, le traitement fait de la cérémonie de déclaration publique de Béré a été de qualité. Le briefing préliminaire dont les journalistes ont pu bénéficier lors du déjeuner de presse a été très positif. 


\section{DIFFICULTES RENCONTREES}

Les principales difficultés rencontrées durant la mise en œuvre du programme sont entre autres :

1. Alors qu'au Sénégal, TOSTAN disposait localement des ressources humaines locales nécessaires pour la mise en œuvre du programme, au Burkina, MWANGAZA a dû recruter des facilicateurs communautaires ayant la capacité de maîtriser le contenu du curriculum en dehors des villages où les programmes allaient se dérouler. En recrutant ainsi hors de la communauté, MWANGAZA a dû faire face à des coûts supplémentaires non prévues au départ.

2. Un retard dans le démarrage des activités de formation, dû essentiellement aux procédures de signature des contrats avec les institutions de financement du programme a été observé. Prévue pour être dispensée en une seule période continue, le programme éducatif a finalement débuté avec quatre mois de retard, et par conséquent elle a été étalée sur deux campagnes (deux saisons sèches).

3. Il y a eu le départ de certains facilitateurs après la première campagne dont la raison principale demeure la recherche d'un emploi meilleur. Il a fallu les remplacer par d'autres personnes afin d'assurer la poursuite des activités.

4. Les événements sociaux (baptêmes, mariages, funérailles...) qui sont survenus pendant le déroulement des classes ont entraîné des cycles de reports et de rattrapages des séances. Cette situation est à l'origine de plusieurs déconvenues observées par les facilitateurs dans la préparation de quelques séances.

5. L'absence des mesures d'accompagnement pour soutenir et motiver les participants au programme. Évoquée à plusieurs reprises par ces auditeurs, cette absence des mesures d'accompagnement a entraîné le manque d'engouement de certains auditeurs qui s'est traduit par les irrégularités dans la participation aux séances.

\section{LEÇONS / ENSEIGNEMENTS}

A la lumière de ces résultats, il convient de tirer quelques enseignements sur l'engagement de la communauté pour la promotion de la santé de la reproduction et des actions de développement.

\section{Requérir l'avis des leaders avant toute intervention}

La présentation du programme aux leaders de chaque communauté demeure la première étape de la mise en œuvre du programme d'éducation à base communautaire. Cette action a pour but d'obtenir l'adhésion de ces responsables. Grâce à cette démarche, le soutien de ces personnes ressources a été très déterminant dans la mobilisation des populations tout le temps que le programme duré. 


\section{Un stage d'immersion pour tous les agents du programme}

Dans le cadre de la mise en œuvre du programme d'éducation à base communautaire, Mwangaza a fait appel à de jeunes gens (facilitateurs communautaires) pour l'exécution des activités d'appui aux communautés.

Ces facilitateurs communautaires étaient pour la plupart à leur première expérience de travail avec des communautés villageoises. Ils ont passé la majeure partie de leur vie dans les grands centres urbains. Un apprentissage, une adaptation est donc indispensable pour eux, pour leur permettre d'être opérationnels au niveau village.

C'est une occasion qui a été offerte au personnel de se familiariser avec

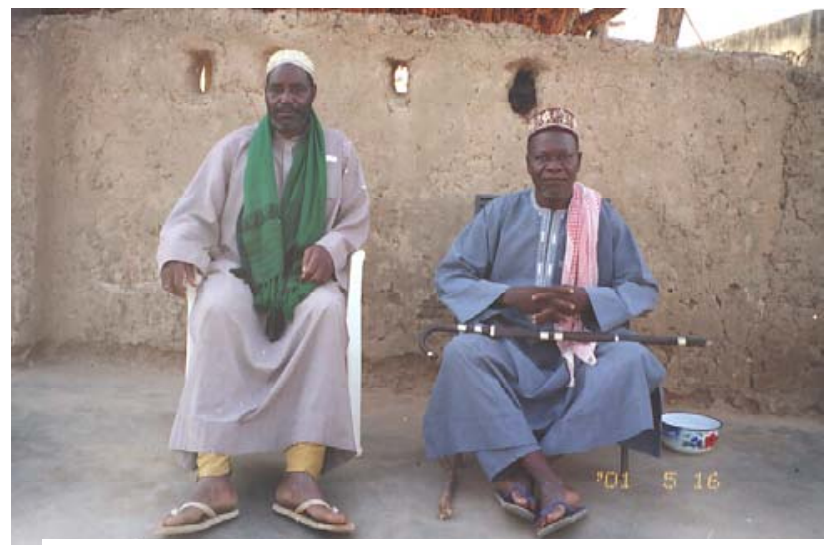

Leaders communautaires dont le soutien a été déterminant dans la mise ouvre du programme. la communauté afin de réapprendre un certain nombre de valeurs comme la confiance, le respect mutuel, la simplicité, l'acceptation des autres tel qu'ils sont etc. Ces valeurs sont très importantes et aident dans le travail au sein des communautés. Elles constituent la pierre angulaire de la philosophie de Mwangaza.

\section{W Vivre au village pour instaurer la confiance}

Une des philosophies du programme est d'habiter dans le village pour être plus près des personnes avec lesquelles on travaille. Ainsi, l'ensemble des membres de l'équipe de mise en œuvre (les 23 facilitateurs et les 3 superviseurs) est resté dans les villages durant tout le temps du programme. Ce séjour dans les villages a permis d'instaurer une confiance entre la communauté et les agents du programme. Le personnel du programme et la communauté ont appris à se connaître, à se comprendre et à parler le même langage.

\section{Utiliser la langue et le langage des villageois}

La langue de travail dans la zone du programme est le mooré. Les facilitateurs communautaires qui ont été recrutés étaient soit des mossis ou des gens qui s'exprimaient assez aisément dans cette langue. Afin de mettre tout le monde au même niveau et les rendre opérationnels, un séminaire sur la transcription du mooré a été organisé. Ce séminaire qui a duré une semaine, a permis aux facilitateurs de maîtriser les règles de transcription du mooré, d'améliorer leur vocabulaire surtout en ce qui concerne les thèmes du paquet pédagogique.

Cette action a été bénéfique d'autant que le programme est perçu comme une stratégie de post alphabétisation. Ainsi les facilitateurs s'exprimaient et écrivaient en mooré. Ce qui a 
motivé les participants déjà alphabétisés à s'exprimer et à susciter l'envie d'apprendre à lire, à écrire et à compter chez tous les participants qui n'avaient pas cette compétence.

Les participants (auditeurs et auditrices) qui avaient déjà été alphabétisés prenaient des notes durant l'animation des séances. Nous avons même observé que certains participants en s'absentant, prenaient soins de remettre leurs cahiers de notes à un autre pour qu'il lui note tout ce qui se passera au cours de la séance. Du coup il y a eu des engagements ferme chez ceux qui n'étaient pas compétent dans l'écriture, le calcul et la lecture d'aller s'inscrire dans un centre d'alphabétisation.

\section{Apprentissage par expérience}

La démarche pédagogique qui a été appliquée consiste à alterner les sessions de formation et la pratique des apprentissages sur le terrain. Cela signifie que le contenu de l'ensemble des quatre modules n'a pas été enseigné aux facilitateurs communautaires au cours d'une même session de formation.

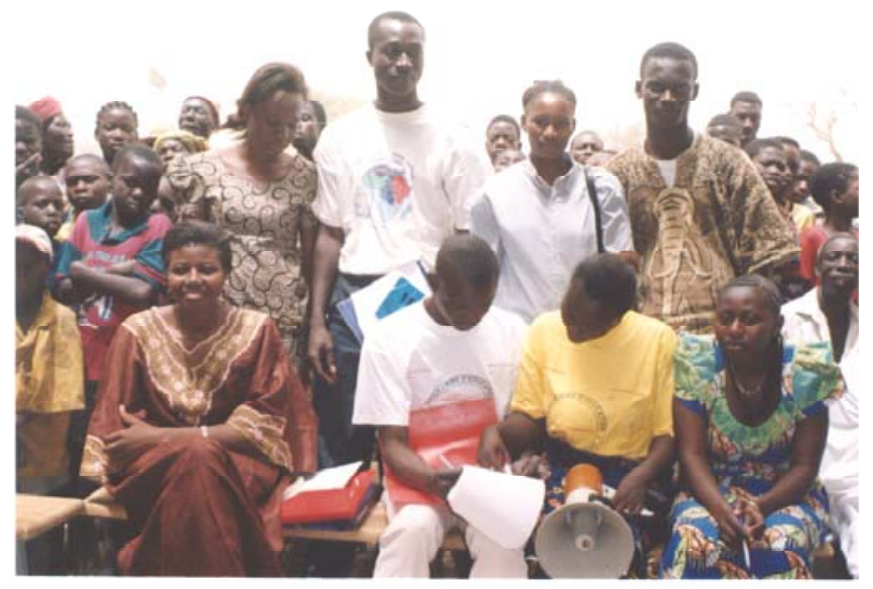

Les facilitateurs communautaires lors de la rencontre inter-village à Bindé.

Cette démarche itérative veut que les facilitateurs acquièrent des compétences, les appliquent, tirent les leçons de cette application avant d'acquérir d'autres compétences. Basée sur le modèle de l'apprentissage par l'expérience, elle permet de corriger et d'élaborer des stratégies pour la suite de l'intervention.

Ainsi les facilitateurs ont d'abord été formés sur le contenu des modules d'éducation aux Droits Humains, résolution des problèmes et sur les techniques d'animation. Après la formation qui a duré trois semaines, les facilitateurs ont, chacun rejoint son village d'affectation pour l'animation des séances.

Après les évaluations intervenues à la fin de la première campagne, un autre séminaire de formation, sur le contenu des modules hygiène et prévention de la maladie et santé de la femme a été organisé.

Tout ceci a permis de s'assurer que les apprentissages aussi bien chez les facilitateurs que chez les participants sont maîtrisés avant de poursuivre la formation des derniers modules.

\section{Au moins 18 mois pour avoir des résultats}

Initialement le programme était prévu pour durer seulement sept mois, soit une saison sèche. Dans le démarrage des activités du programme, un retard a été accusé. Ce retard a 
créé les conditions défavorables à la mise en œuvre des activités dans les délais impartis, ce qui a entraîné l'extension du temps d'exécution des activités et une répercussion sur les coûts. Cette extension a été déterminante dans la dynamique sociale que nous avons observée dans la communauté. En effet, entre les deux saisons sèches, les populations ont « assimilé » tout ce qui a été dit au cours de l'animation des séances. Aussi des activités de suivi accompagnement des communautés ont-elles été développées.

Durant cette période verte, le programme est resté en contact avec la population par l'intermédiaire des superviseurs. Les superviseurs ont accompagné les villages dans la mise en œuvre des plans d'action qui ont été élaborés dans les classes. Le changement de comportement est une œuvre de longue haleine.

\section{Chaque communauté désigne ceux qui vont bénéficier du " savoir »}

Une des responsabilités de chaque communauté villageoise, c'est la désignation des personnes devant prendre part aux activités du programme. Chaque village a élaboré les critères de désignation des participants (auditrices et auditeurs) au programme. Le programme a respecté la décision de chaque communauté conformément à sa philosophie de respect des décisions prises par ses partenaires.

\section{W Des séances publiques hors des classes pour toute la population}

Certaines séances animées en classe ont été jugées assez importantes et dignes d'intérêt. Les participants au programme ont demandé que ces séances soient animées hors de la classe pour toute la population du village. Les séances suivantes ont été animées lors des séances publiques : 1'excision, l'espacement des naissances, les IST/VIH/SIDA, la sexualité, la préparation à l'accouchement. Ces séances animées hors de la classe ont attiré l'attention des hommes et des femmes du village sur la nécessité d'entreprendre des actions pour la préservation de la santé de la population. Vu le succès remporté par ces animations. Une telle pratique a été encouragée dans tous les villages.

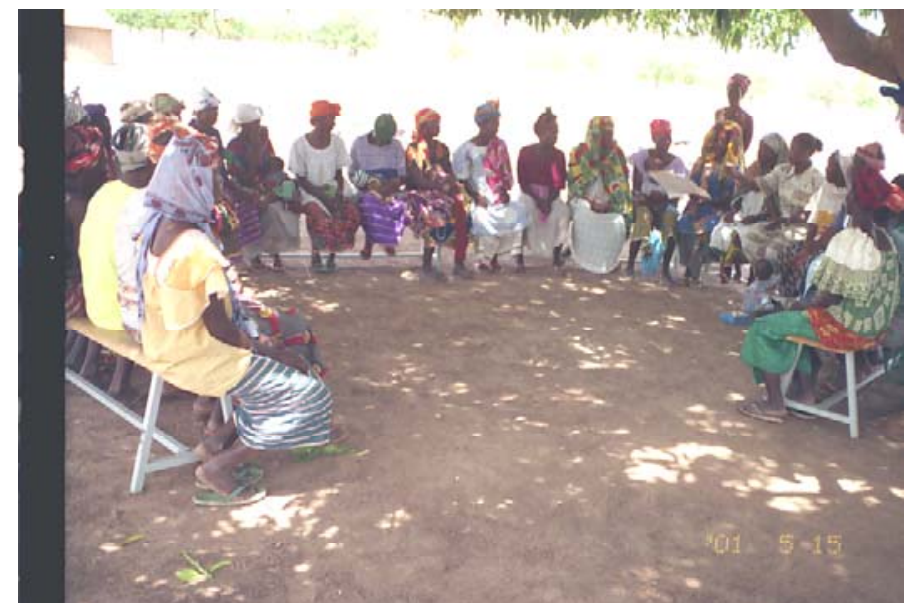

Séance éducative sur les Droits Humains avec les auditrices de. Yoroho

\section{Méthodes actives pour l'animation des séances}

La plupart des participants au programme étaient des adultes. Travailler avec des adultes implique l'utilisation de méthodes qui leur sont appropriées. Pour cela les méthodes actives d'éducation ont été utilisées. Les caractéristiques générales de ces méthodes actives d'éducation sont entre autres : 
1. Activité des sujets à instruire. C'est l'application du principe selon lequel le sujet apprend mieux s'il est engagé personnellement dans l'action ;

2. Motivation des sujets à instruire. La motivation doit être intrinsèque, c'est à dire que les sujets se sentent concernés, impliqués.

3. Le moniteur est plutôt un facilitateur, un catalyseur, qu'un instructeur au sens strict. Son autorité change de nature et d'expression.

4. Le contrôle comme tel disparaît, du moins dans sa forme classique de vérification du savoir intellectuel ou pratique. Il devient une auto-évaluation des individus ou des groupes.

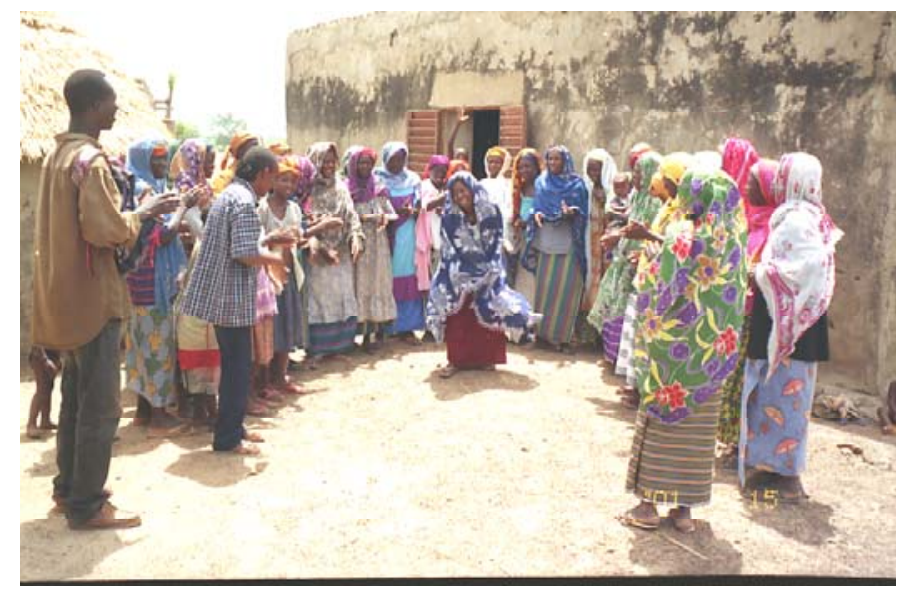

Les auditrices de Kondrin esquissent des pas de danse à la fin d'une séance, en présence des facilitateurs et du superviseur

C'est dans cette ambiance que le programme a été conduit. Les techniques participatives suivantes ont été utilisées pour permettre une réelle adhésion et une implication optimale : chant, danse, théâtre, travaux de groupes. Cela permet aux participants de retenir l'essentiel de ce qu'ils apprennent.

\section{W Dix séances par mois sont suffisantes pour soutenir l'action}

Nous avons aussi remarqué qu'en animant au plus 3 séances par semaine, donc pas plus de 10 séances dans le mois, les participants avaient le temps pour planifier et pour mettre en œuvre des activités au sein de la communauté. Ces dispositions ont été très bien acceptées par la communauté, qui arrivait à bien mener certaines activités personnelles ou collectives sans contraintes majeures.

\section{L'éducation aux Droits Humains comme fondation du bâtiment}

Le premier module mis en œuvre, dans le cadre du paquet pédagogique, a été l'éducation aux Droits Humains. Cet enseignement a créé un environnement favorable à la discussion avec la population sur tous les sujets en rapport avec leur quotidien. Ces connaissances ont contribué à développer au sein des communautés une culture de l'action. 


\section{CONCLUSION}

Le programme d'éducation à base communautaire se veut le développement de stratégies à l'échelle communautaire visant à promouvoir les conditions d'une qualité de vie supérieure pour un nombre plus élevé de population de la zone d'intervention.

Au terme d'une telle expérience, fruit de la collaboration entre l'ONG TOSTAN, MWANGAZA ACTION et des partenaires au développement, il faut retenir de cette expérience quatre modules pertinents dont l'enseignement a connu un impact réel sur les villages du programme : les Droits Humains, le processus de résolution des problèmes, l'hygiène et prévention de la maladie et la santé de la femme. Le niveau d'implication, ainsi que l'engouement manifesté par les populations dans la mise en œuvre de ce programme illustrent à merveille l'intérêt que revêt cette expérience.

En effet, l'enseignement des Droits Humains et de la santé de la reproduction, fondement de ce programme, a révolutionné le quotidien des populations, par l'approche participative et active qui a caractérisé l'enseignement des modules.

L'apport du programme s'observe principalement dans les différentes actions et engagements entrepris par les communautés en termes d'activités de développement, de salubrité, de promotion de la santé et des Droits Humains. C'est une prise de conscience qui est perceptible au niveau de tous les acteurs communautaires. Toutes choses qui augurent de la bonne appropriation du programme par les populations.

Dans la mise en œuvre de ce programme, faut-il le rappeler, la participation communautaire demeure une démarche inéluctable tant elle constitue un cadre adéquat de réflexion objective sur les problèmes en vue de leur trouver des solutions idoines.

L'action de Droits Humains, soutenue par l'enseignement des séances sur la santé de la reproduction, a contribué à délivrer les femmes des pesanteurs culturelles dont elles sont parfois victimes. L'idée d'une promotion du genre par la prise en compte des femmes dans les décisions et la lutte contre les pratiques néfastes à la santé dont elles sont victimes semble faire son chemin. La solidarité sociale se solidifie entre les membres d'une même communauté et entre les villages voisins.

En dépit des difficultés rencontrées pendant la mise en l'œuvre de ce programme, nous pouvons observer une nette amélioration des conditions sociales de populations de la zone d'intervention.

Cependant, comme tout processus de changement, des résistances ont été observées dans la mise en œuvre de ce programme. Loin d'être de véritables obstacles, celles-ci doivent plutôt aider à mieux formuler et nourrir les principaux enseignements à tirer de cette expérience. Des enseignements qui serviront à réajuster efficacement les démarches dans le cadre de la réplication éventuelle de ce programme. 
«Prévenir, c'est à la fois " devancer, aller au devant de » et « avertir de ». La déclaration publique d'abandon de la pratique de l'excision de Béré, qui a été le moment fort de cette expérience, s'inscrit dans le cadre de la prévention sociale.

Par cela les populations de Béré et de Bindé s'engagent à refuser et à dénoncer dans leur contrée toute pratique allant à l'encontre de la promotion du droit et de la santé de la femme comme l'excision.

L'acte posé par la population de Béré et de Bindé est un message à l'endroit de tous ceux qui sont encore hésitants à abandonner la pratique de l'excision, pour leur dire « qu'ils trouveront sur leur route des fem mes et des hommes résolument engagés pour l'abandon de la pratique de l'excision. Outre ces femmes et hommes, ils rencontreront également les ancêtres (acte posé par le représentant des chefs coutumiers) qui s'opposeront à eux ».

La déclaration publique en tant que facteur de changement est à encourager dans le cadre de la lutte contre la pratique de l'excision. 